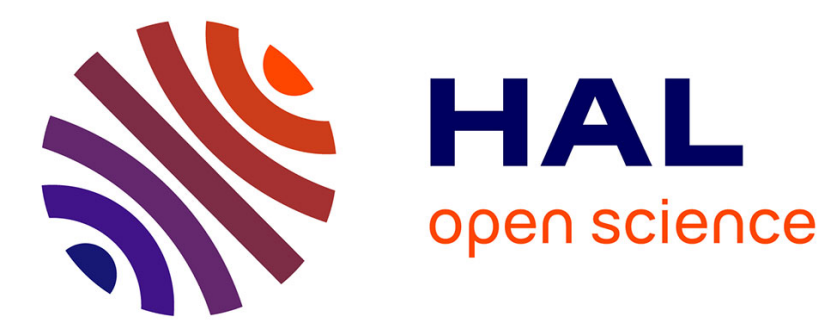

\title{
Approximation of general arch problems by straight beam elements
}

\author{
Michel Bernadou, Y. Ducatel
}

\section{To cite this version:}

Michel Bernadou, Y. Ducatel. Approximation of general arch problems by straight beam elements. RR-0120, INRIA. 1982. inria-00076440

\section{HAL Id: inria-00076440 \\ https://hal.inria.fr/inria-00076440}

Submitted on 24 May 2006

HAL is a multi-disciplinary open access archive for the deposit and dissemination of scientific research documents, whether they are published or not. The documents may come from teaching and research institutions in France or abroad, or from public or private research centers.
L'archive ouverte pluridisciplinaire HAL, est destinée au dépôt et à la diffusion de documents scientifiques de niveau recherche, publiés ou non, émanant des établissements d'enseignement et de recherche français ou étrangers, des laboratoires publics ou privés. 


\section{APPROXIMATION OF GENERAL ARCH \\ PROBLEMS BY STRAIGHT BEAM ELEMENTS, *}

M. BERNADOU ( $\left.{ }^{1}\right)-Y$. DUCATEL (2)

RESUME : Dans ce rapport, nous approchons la solution d'un problème de déformation d'un arc de forme quelconque par une méthode non conforme utilisant des éléments finis plats et prenant en compte l'intégration numérique. Nous donnons les conditions de compatibilité qui doivent être satisfaites aux noeuds du maillage et qui assurent pour cette méthode le même ordre de convergence que pour les méthodes classiques d'éléments finis conformes.

ABSTRACT : In this report, we approximate the solution of a problem of a general arch by a nonconforming method using straight beam elements and taking into account numerical integration. Compatibility conditions which have to be satisfied at the mesh points are given. These conditions ensure for this method the same order of convergence as usual conforming finite element methods.

( $\left.{ }^{1}\right)$ I.N.R.I.A., Domaine de Voluceau - Rocquencourt - B.P. 105 78153 LE CHESNAY CEDEX.

(2) Université de Poitiers, Département de Mathématiques 40, Avenue du Recteur Pineau - 86022 POITIERS CEDEX.

Classification Math. Reviews : 65N30; 73KO5; 73K15; 73K25.

* "submitted to Numerische Mathematik" 
The purpose of this paper is to extend to the case of a general arch the studies of CIARLET [5], JOHNSON [14] and KIKUCHI [16] concerning the particular case of a circular arch.

For solving the general arch problem, we intend to analyze a nonconforming method using straight beam elements. So, we introduce

* a nonconforming approximation of the geometry of the mean line of the arch using straight segments ;

** a pseudo-conforming approximation of the components of the displacement, i.e., an approximation using conforming beam elements over every straight segment.

Then, the connection between degrees of freedom attached to the same mesh point is obtained through the compatibility conditions. They insure the consistency between exact and approximated bilinear and linear forms and they are crucial in proving the convergence of this method.

Such methods can be generalized to thin shell. We refer for example to the works of CLOUGH-JOHNSON [8,9] or DAWE [10] for a description as well as some numerical experiments and to BERNADOU-DUCATEL [4] for corresponding numerical analysis.

Let us also mention the work of ISHIHARA [13] for solving eigenvalue problems of circular arches and the one of GELLERT-LAURSEN [12] who propose a mixed finite element method. 
1 - Formulation of the arch problem deduced from thin shell theory of W.T. KOITER

1.1. Some notations from thin shell theory.

1.2. Variational formulation of the general arch problem.

2 - A conforming finite element method

2.1. The finite element space $\vec{V}_{h}$.

2.2. The discrete problem.

3 - The discrete problem using straight beam elements; compatibility relations

3.1. The approximate $\operatorname{arch} a_{h}$.

3.2. The discrete space $\overrightarrow{\widetilde{V}}_{h}$ using compatibility conditions.

3.3. The discrete problem.

4 - Convergence and error estimates

4.1. Abstract error estimate.

4.2. Estimate of the consistency error $\left|a\left(\vec{v}_{h}, \vec{w}_{h}\right)-b_{h}\left(\vec{v}_{h}, \vec{w}_{h}\right)\right|$.

4.3. Estimate of the consistency error $\left|f\left(\vec{w}_{h}\right)-f_{h}\left(\vec{w}_{h}\right)\right|$.

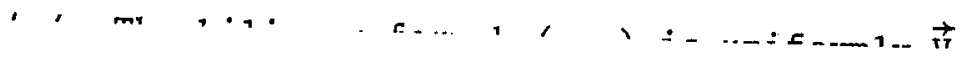

- 1 1 :-+:

- Notations -

We use the same notations as [5]. 


\section{1 - FORMULATION OF THE ARCH PROBLEM DEDUCED FROM THIN SHELL THEORY OF W.T. KOITER}

We consider the mean line $a$ of a general arch as the normal cross section of an infinite cylinder. In linear theory, if the loads are perpendicular to the axis of the cylinder, the component of the displacement along the axis of the cylinder is identically equal to zero, so that the shell deformation problem is reduced to a one dimensional problem set along the generating line of the cylinder.

The reader who is not familiar with thin shell theory can directly go to section 1.2 .

\section{1 - Some notations from thin shell theory :}

Let $\Omega=] \circ, L[x]-\infty,+\infty[$ be an open subset in a plane $\delta^{2}$ and let $\delta^{3}$ be the usual Euclidean space with its orthonormal reference system $\left(0, \vec{e}_{1}, \vec{e}_{2}, \vec{e}_{3}\right)$.

The cylinder $C$ is the image of the set $\bar{\Omega}$ by the following mapping $\vec{\phi}=\phi^{i} \vec{e}_{i}, \vec{\phi}: \vec{\Omega} \subset \delta^{2} \rightarrow \delta^{3} \quad$ (see Fig. 1.1.1) :

$$
\vec{\phi}:\left(\xi^{1}, \xi^{2}\right) \in \bar{\Omega} \longrightarrow\left\{\begin{array}{l}
x=\phi^{1}\left(\xi^{1}\right) \\
y=\phi^{2}\left(\xi^{1}\right) \\
z=\xi^{2}
\end{array}\right.
$$

Let $Q$ be the trace of the cylinder in the plane $z=0$. Here and subsequently, we shall constantly identify the arch with its middle line.

As a rule, we shall use Greek letters for indices which take their values in the set $\{1,2\}$ while Latin letters will be used for indices which take their values in the set $\{1,2,3\}$. For these indices, we shall also use Einstein's convention for summation. 


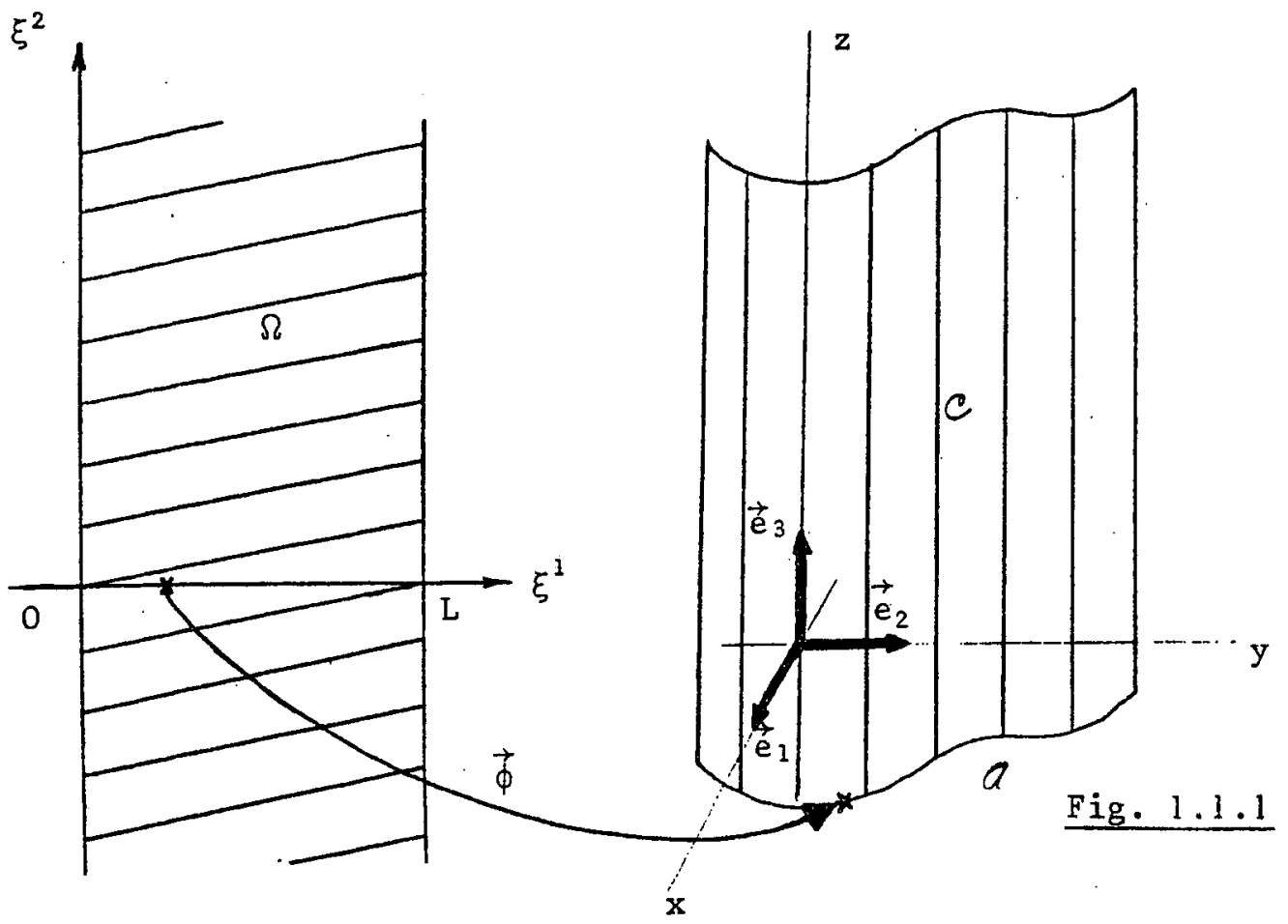

With usual notations of shell theory - see $[17,18]$

for instance - , the covariant basis is given by

$$
\begin{gathered}
\vec{a}_{1}=\vec{\phi}_{, 1}=\left\{\begin{array}{c}
\left(\phi^{1}\right) ! \\
\left(\phi^{2}\right) \\
0
\end{array} \quad \vec{a}_{2}=\vec{\phi}, 2=\left\{\begin{array}{l}
0 \\
0=\vec{e}_{3} \\
1
\end{array}\right\}\right. \\
\vec{a}_{3}=\frac{1}{\sqrt{a}}\left\{\begin{array}{c}
\left(\phi^{2}\right)^{\prime} \\
-\left(\phi^{1}\right) \\
0
\end{array}\right\}
\end{gathered}
$$

where we denote

- $a=\left(\left(\phi^{1}\right)^{\prime}\right)^{2}+\left(\left(\phi^{2}\right)^{\prime}\right)^{2}$.

Subsequently, we shall assume that

$$
\left.\phi_{E C^{3}(\bar{I})}^{\alpha} ; I=\right]_{0, L[},
$$

and that all the points of the surface are regular, i.e.,

$$
\vec{a}_{1} \times \vec{a}_{2} \neq \vec{o}
$$


The first fundamental form $\left(a_{\alpha \beta}\right)$ of the surface is defined by

$$
a_{\alpha \beta}=\vec{a}_{\alpha} \cdot \vec{a}_{\beta}
$$

so that

$$
a_{11}=a \quad ; \quad a_{12}=a_{21}=0 \quad ; \quad a_{22}=1 \cdots \quad(1.1 .7)
$$

The inverse matrix $\left(a^{\alpha \beta}\right)$ of the matrix $\left(a_{\alpha \beta}\right)$ is such that

$$
a^{11}=\frac{1}{a} \quad ; \quad a^{12}=a^{21}=0 ; a^{22}=1 .
$$

Then, to the covariant basis $\left(\vec{a}_{i}\right)$, we associate the contravariant basis $(\vec{a})$ of the surface $C$ given by

$$
\vec{a}^{\alpha}=a^{\alpha \beta} \vec{a}_{\beta} \text {. }
$$

i.e.,

$$
\vec{a}^{1}=\frac{1}{a} \vec{a}_{1} \quad ; \quad \vec{a}^{2}=\vec{a}_{2} ; \vec{a}^{3}=\vec{a}_{3} \text {. }
$$

The second fundamental form $\left(b_{\alpha \beta}\right)$ of the surface is given by

$$
b_{\alpha \beta}=b_{\beta \alpha}=-\vec{a}_{\alpha} \cdot \vec{a}_{3, \beta}=\vec{a}_{3} \cdot \vec{a}_{\alpha, \beta}
$$

and

$$
b_{\alpha}^{\beta}=a^{\beta \lambda} b_{\alpha \lambda}
$$

i.e.,

$$
\left\{\begin{array} { l } 
{ b _ { 1 1 } = - \frac { a } { R } } \\
{ b _ { 1 2 } = b _ { 2 1 } = b _ { 2 2 } = 0 }
\end{array} \quad \left\{\begin{array}{l}
b_{1}^{1}=-\frac{1}{R} \\
b_{2}^{1}=b_{1}^{2}=b_{2}^{2}=0
\end{array}\right.\right.
$$


where $\mathrm{R}$ denotes the curvature radius, i.e.,

$$
\frac{1}{\mathrm{R}}=\frac{\left(\phi^{1}\right)^{\prime}\left(\phi^{2}\right)^{\prime \prime}-\left(\phi^{1}\right)^{\prime \prime}\left(\phi^{2}\right)^{\prime}}{\mathrm{a}^{3 / 2}} .
$$

The expression of Christoffel symbols $\left(\Gamma_{\beta \gamma}^{\alpha}\right)$ of the surface is

$$
\Gamma_{B \gamma}^{\alpha}=\vec{a}^{\alpha} \cdot \vec{a}_{\gamma, \beta}
$$

so that

$$
\begin{aligned}
& \Gamma_{11}^{1}=\frac{\left(\phi^{2}\right)^{\prime}\left(\phi^{1}\right)^{\prime \prime}+\left(\phi^{2}\right)^{\prime}\left(\phi^{2}\right)^{\prime \prime}}{a} ; \\
& \Gamma_{12}^{1}=\Gamma_{21}^{1}=\Gamma_{22}^{1}=\Gamma_{11}^{2}=\Gamma_{12}^{2}=\Gamma_{21}^{2}=\Gamma_{22}^{2}=0 .
\end{aligned}
$$

From now on, we assume

Hypothesis 1.1.1: The loads applied to the cylinder are perpendicular to the axis of the cylinder and independent of $\xi^{2}$.

In the following, the unknowns of the problem are the three functions

$$
u_{1}=u_{1}\left(\xi^{1}\right) \quad, u_{2} \equiv 0, u_{3}=u_{3}\left(\xi^{1}\right)
$$

which represent the covariant components of the displacement $\vec{u}=\vec{u}\left(\xi^{l}\right)$ of the point $\vec{\phi}\left(\xi^{1}\right)$, i.e., $\vec{u}=u_{i} \vec{a}_{i}$. The results (1.1.16) are consequence of Hypothesis 1.1 .1 .

To any displacement field $\vec{v}=v_{i} \vec{a}_{i}$, we associate the strain tensor $\left(\gamma_{\alpha \beta}\right)$ and the change of curvature tensor $\left(\rho_{\alpha \beta}\right)$ through the relations

$$
\begin{aligned}
\gamma_{\alpha \beta}(\vec{v})= & \frac{1}{2}\left(v_{\beta \mid \alpha}+v_{\alpha \mid \beta}\right)-b_{\alpha \beta} v_{3}, \\
\rho_{\alpha \beta}(\vec{v})= & v_{3 \mid \alpha \beta}+\frac{1}{4} b_{\alpha}^{\lambda}\left(3 v_{\lambda \mid \beta}-v_{\beta \mid \lambda}\right)+\frac{1}{4} b_{\beta}^{\lambda}\left(3 v_{\lambda \mid \alpha}-v_{\alpha \mid \lambda}\right)+ \\
& +\left.b_{\alpha}^{\lambda}\right|_{\beta} v_{\lambda},
\end{aligned}
$$




$$
\gamma_{\beta}^{\alpha}(\vec{v})=a^{\alpha \lambda} \gamma_{\lambda \beta}(\vec{v}) \quad, \quad \rho_{\beta}^{\alpha}(\vec{v})=a^{\alpha \lambda} \rho_{\lambda \beta}(\vec{v})
$$

i.e.,

$$
\left.\begin{array}{l}
\gamma_{1}^{1}(\vec{v})=\frac{1}{\sqrt{a}}\left\{\left(\frac{v^{2}}{\sqrt{a}}\right)^{\prime}+\frac{\sqrt{a}}{R} v_{3}\right\}, \gamma_{2}^{1}(\vec{v})=\gamma_{1}^{2}(\vec{v})=\gamma_{2}^{2}(\vec{v})=0, \\
\rho_{1}^{1}(\vec{v})=\frac{1}{\sqrt{a}}\left(\frac{1}{\sqrt{a}}\left(v_{3}^{\prime}-\frac{1}{R} v_{1}\right)\right)^{\prime}, \rho_{2}^{1}(\vec{v})=\rho_{1}^{2}(\vec{v})=\rho_{2}^{2}(\vec{v})=0 .
\end{array}\right\}
$$

\section{2 - Variational formulation of the general arch problem :}

For simplicity; we assume that the arch is clamped. Then the admissible displacement space $\vec{V}$ is defined by

$$
\overrightarrow{\mathrm{v}}=\left\{\overrightarrow{\mathrm{v}}=\left(\mathrm{v}_{1}, \mathrm{v}_{3}\right) \in \mathrm{H}_{0}^{1}(\mathrm{I}) \times \mathrm{H}_{0}^{2}(\mathrm{I})\right\}
$$

where we denote $I=] 0, \mathrm{~L}[$.

Equipped with the scalar product

$$
((\vec{u}, \vec{v}))=\left(\left(u_{1}, v_{1}\right)\right)_{1, I}+\left(\left(u_{3}, v_{3}\right)\right)_{2, I},
$$

the space $\vec{V}$ is a Hilbert space. The corresponding norm is denoted

$$
\|\vec{v}\|=[((\vec{v}, \vec{v}))]^{1 / 2} .
$$

For clarity and without loss of generality, we assume that the arch is elastic, homogeneous and isotropic. Then, using the definition (1.1.17) of the strain tensor and of the change of curvature tensor, the strain energy of the arch $\frac{1}{2} a(\vec{v}, \vec{v})$ is abtained from

$$
a(\vec{u}, \vec{v})=\int_{0}^{L} \frac{E e}{1-v^{2}}\left\{\gamma_{1}^{1}(\vec{u}) \gamma_{1}^{1}(\vec{v})+\frac{e^{2}}{12} \rho_{1}^{1}(\vec{u}) \rho_{1}^{1}(\vec{v})\right\} \sqrt{a} d \xi
$$

where e,E $(E .>0)$ and $V\left(0^{\circ}<V^{\circ}<\frac{1}{2}\right)$ denote respectively the thickness, the Young modulus and the Poisson coefficient of the arch. Moreover the expressions $\gamma_{1}^{1}(\vec{v}), \rho_{1}^{1}(\vec{v})$ are given by $(1.1 .17)$ and we have set $\xi^{1}=\xi$. 
The potential energy of exterior forces is given by $f(\vec{v})=\int_{0}^{L} \vec{p} \cdot \vec{v} \sqrt{a} d \xi=\int_{0}^{L}\left(p^{1} v_{1}+p^{3} v_{3}\right) \sqrt{a} d \xi \quad(1.2 .4$.

where the functions $p^{i}$ are the contravariant components over the basis $\left(\vec{a}_{i}\right)$ of the density per unit surface of the exterior forces applied to the arch.

Then, the arch problem admits the following variational formulation : Find $\vec{u} \in \vec{V}$ such that

$$
a(\vec{u}, \vec{v})=f(\vec{v}), \forall \vec{v} \in \vec{v}
$$

where the forms $a(.,$.$) and f($.$) are defined by (1.2 .3)$ and $(1.2 .4)$ respectively.

Theorem 1.2.1.: The problem (1.2.5) admits one and only one solution. Proof : Similar to the proof of [5, Theorem 8.1.2].

\section{2 - A CONFORMING FINITE ELEMENT METHOD}

In this paragraph, we only consider an approximation of the displacement using a conforming finite element method. This stage will be a useful intermediary for the analysis of an appro-. ximation using straight beam elements.

\section{1 - The finite element space $\vec{V}_{h}$ :}

Consider a regular partition $\mathfrak{b}_{h}$ of the interval $\overline{\mathrm{I}}$, i.e., $\bar{I}=[0, L]=\bigcup_{i=1}^{M_{h}} k_{i}$, with $k_{i}=\left[\xi_{i-1}, \xi_{i}\right], 1 \leq i \leq M_{h}$,

such that,

$$
h=\max _{1 \leq i \leq M_{h}} h_{K_{i}} \text { approaches zero, }
$$


where $M_{h}$ is a strictly positive integer and $h_{K_{i}}=$ meas $\left(K_{i}\right)$. To each partition, we associate a product of finite element spaces $\overrightarrow{\mathrm{x}}_{\mathrm{h}}=\mathrm{x}_{\mathrm{h}_{1}} \times \mathrm{x}_{\mathrm{h}_{2}}$ defined as follows :

Space $X_{h_{1}}$ is the finite element space whose functions are such that :

(i) on every $K_{i}, 1 \leq i \leq M_{h}$, they belong to $P_{1}\left(K_{i}\right)$;

(ii) on every $k_{i}, 1 \leq i \leq M_{h}$, they are determined by their (2.1. values at the extremities of $k_{i}$;

(iii) $x_{h_{1}} \subset C^{0}(\bar{I})$.

Space $\mathrm{X}_{\mathrm{h}_{2}}$ is the finite element space whose functions are such that :

(i) on every $K_{i}, 1 \leq i \leq M_{h}$, they belong to $\mathrm{P}_{3}\left(\mathrm{~K}_{i}\right)$;

(ii) on every $\mathrm{K}_{i}, 1 \leq i \leq \mathrm{M}_{\mathrm{h}}$, they are determined by their $(2.1$. values and by the values of their derivatives at the extremities of $\mathrm{k}_{i}$;

(iii) $x_{h_{2}} \subset c^{1}(\bar{I})$.

Next, we define a subspace $\vec{v}_{h}=v_{h_{1}} \times v_{h_{2}}$ of $\vec{x}_{h_{1}}$ taking into account the boundary conditions which appear in the definition of the space $\vec{V}$.

$$
\begin{aligned}
& \text { More precisely, } \\
& \mathrm{v}_{\mathrm{h}_{1}}=\left\{\mathrm{v}_{\mathrm{h}} \in \mathrm{X}_{\mathrm{h}_{1}} ; \mathrm{v}_{\mathrm{h}}(0)=\mathrm{v}_{\mathrm{h}}(\mathrm{L})=0\right\} \\
& \mathrm{v}_{\mathrm{h}_{2}}=\left\{\mathrm{v}_{\mathrm{h}} \in \mathrm{X}_{\mathrm{h}_{2}} ; \mathrm{v}_{\mathrm{h}}(0)=\mathrm{v}_{\mathrm{h}}^{\prime}(0)=\mathrm{v}_{\mathrm{h}}(\mathrm{L})=\mathrm{v}_{\mathrm{h}}^{\prime}(\mathrm{L})=0\right\},
\end{aligned}
$$

so that

$$
\overrightarrow{\mathrm{v}}_{\mathrm{h}} \in \overrightarrow{\mathrm{V}}
$$

hence the name : conforming element method. 


\section{2 - The discrete problem :}

The approximate solution $\vec{u}_{h}^{*}$ is such that

$$
\vec{v}_{h} \in \vec{v}_{h}, \quad a\left(\vec{u}_{h}^{*}, \vec{v}_{h}\right)=f\left(\vec{v}_{h}\right)
$$

The inclusion $\vec{V}_{h} \subset \vec{V}$ involves the theorem :

Theorem 2.2.1.: The problem (2.2.1) has a unique solution.

Using [5], we find the following error estimate.

Theorem 2.2.2.: If the solution $\vec{u}=\left(u_{1}, u_{3}\right)$ of the problem $(1.2 .5)$ belongs to the space $\vec{V} \cap\left(H^{2}(I) \times H^{3}(I)\right)$, then there exists a constant $C$, independent of $h$, such that

$$
\left\|\vec{u}-\vec{u}_{h}^{*}\right\| \leq \operatorname{Ch}\left\{\left|u_{1}\right|_{2, I}^{2}+\left|u_{2}\right|_{3, I}^{2}\right\}^{1 / 2}
$$

At this stage, the discrete problem is seldom realistic in practice, in the sense that it would lead to exactly integrate any regular functions. To circumvent this difficulty, we shall use in the following paragraphes (i) a non conforming approximation of the geometry, (ii) a numerical integration technique. So doing, we generalize the studies of [5] and [14] relative to circular arches.

\section{3 - THE DISCRETE PROBLEM USING STRAIGHT BEAM ELEMENTS ; COMPATIBILITY RELATIONS}

Now we are going to define the approximate $\operatorname{arch} a_{h}$. Next, we shall introduce the discrete space $\overrightarrow{\widetilde{V}}_{h}$ by using the compatibility conditions. Finally we shall set the discrete problem by using the straight beam elements. 


\section{1 - The approximate arch $a_{\mathrm{h}}$ :}

The (middle line of the) arch $Q$ is defined by the mapping $\vec{\phi}\left(\xi^{1}, 0\right)=\phi^{\alpha}\left(\xi^{1}\right) \vec{e}_{\alpha}$ - see $(1.1 .1)$. In this section, we approximate each component $\phi^{\alpha}, \alpha=1,2$, by its interpolant $\phi_{h}^{\alpha}$ in the finite element space $\Phi_{h}$ defined by

$$
\Phi_{h} \equiv X_{h_{1}}, \quad X_{h_{1}} \text { given by }(2.1 .2)
$$

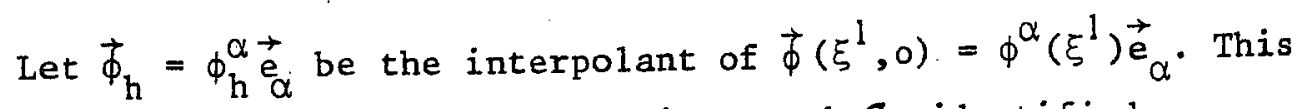
approximation amounts to replace the given $\operatorname{arch} a$, identified with its middle line, by a polygonal arch $a_{h}$. By construction, the images of the mesh points of the reference interval I by the mapping $\vec{\phi}_{h}$ are on the initial arch $\Omega$. These considerations are illustrated by Figure 3.1.1 where we denote $\xi^{1}=\xi$.

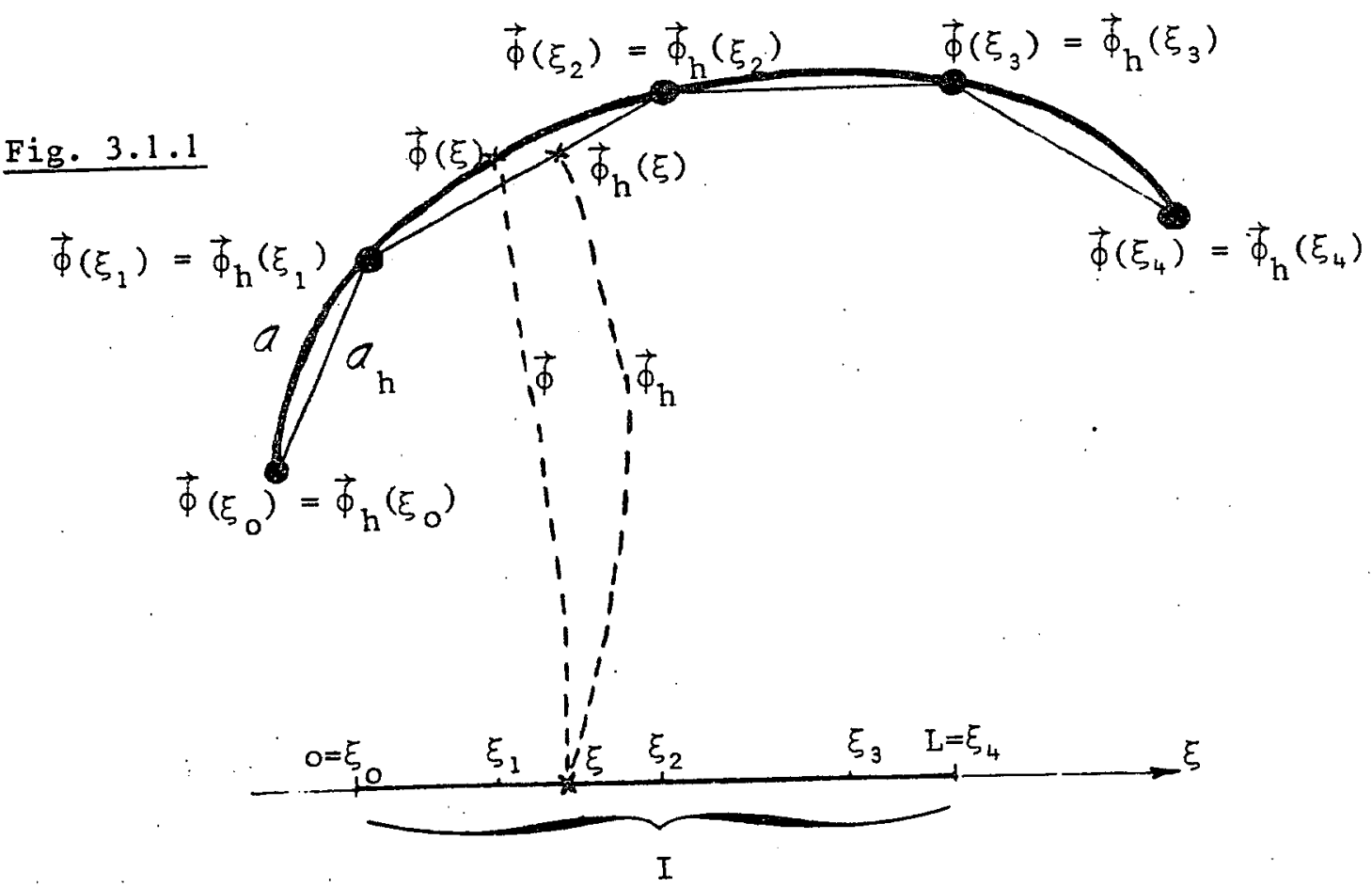

To each straight segment $k_{i}=\vec{\phi}_{h}\left(k_{i}\right), l \leq i \leq M_{h}$, it corresponds a facet of an approximate cylinder - see section 1.1 . Thus, to this facet we are able to associate, by analogy with section 1.1., local basis, fundamental froms, Christoffel symbols, i.e., with obvious notations :

$$
\vec{a}_{h \alpha}=\vec{\phi}_{h, \alpha}, \vec{a}_{h_{3}}=\frac{\vec{a}_{h_{1}} \times \vec{a}_{h_{2}}}{\left|\overrightarrow{a_{h_{1}}} \times \overrightarrow{a_{h_{2}}}\right|},
$$




$$
\begin{aligned}
& \sqrt{a_{h}}=\left|\vec{a}_{h_{1}} \times \vec{a}_{h_{2}}\right| \text {, } \\
& a_{h \alpha \beta}=\vec{a}_{h \alpha} \cdot \vec{a}_{h \beta} \\
& \vec{a}_{h}^{\alpha}=a_{h}^{\alpha \beta} \vec{a}_{h \beta} \text {, where }\left(a_{h}^{\alpha \beta}\right) \text { is the inverse } \\
& \text { matrix of the matrix }\left(a_{h \alpha \beta}\right) \text {, } \\
& \overrightarrow{a_{h}^{3}}=\vec{a}_{h_{3}} \text {. } \\
& b_{h \alpha \beta}=b_{h \alpha}^{\beta}=\Gamma_{h \alpha \beta}^{\lambda}=0 \text {. }
\end{aligned}
$$

It is worth to note that all the previous quantities are constant on any given interval $K_{i}, 1 \leq i \leq M_{h}$, with possible discontinuities at the extremities of $\mathrm{k}_{i}$ due to the discontinuity of the first derivatives of $\vec{\phi}_{h}$ at these points.

More precisely, let us consider a segment $k_{i}$ whose extremities are $\vec{\phi}_{h}\left(\xi_{i-1}\right)=\vec{\phi}\left(\xi_{i-1}\right)$ and $\vec{\phi}_{h}\left(\xi_{i}\right)=\vec{\phi}\left(\xi_{i}\right)$. Then, for any $\xi^{1}=\xi \in\left[\xi_{i-1}, \xi_{i}\right]$, the equation of the corresponding facet of the approximate cylinder is given by

$$
\left.\begin{array}{l}
\vec{\phi}_{h}\left(\xi, \xi^{2}\right)=\left\{(1-\lambda) \phi^{\alpha}\left(\xi_{i-1}\right)+\lambda \phi^{\alpha}\left(\xi_{i}\right)\right\} \overrightarrow{e_{\alpha}}+\xi^{2} \overrightarrow{e_{3}} \\
\text { with } \lambda=\frac{\xi-\xi_{i-1}}{\xi_{i}-\xi_{i-1}},
\end{array}\right\}
$$

so that we obtain

$$
\begin{aligned}
& \overrightarrow{a_{h_{1}}}=\overrightarrow{\phi_{h, 1}}=\frac{\phi^{\alpha}\left(\xi_{i}\right)-\phi^{\alpha}\left(\xi_{i-1}\right)}{\xi_{i}-\xi_{i-1}} \overrightarrow{e_{\alpha}}, \\
& \overrightarrow{a_{h_{2}}}=\overrightarrow{\phi_{h, 2}}=\overrightarrow{e_{3}} .
\end{aligned}
$$

3.2 - The discrete space $\stackrel{\vec{v}}{\mathrm{~h}}_{\mathrm{h}}$ using compatibility conditions :

The main idea of the approximation of the $\operatorname{arch} \emptyset$ by the piecewise straight arch $a_{h}$ is to write the strain energy of the 
approximate $\operatorname{arch} a_{h}$ as a sum of strain energies of "elementary" straight beams.

In this goal, we shall introduce in a first step a discrete space $\widetilde{\mathrm{X}}_{\mathrm{h}}$.

In a second step, in order to get an approximate strain energy which is consistent with the strain energy of the $\operatorname{arch} a$, we shall introduce constraints, i.e., compatibility conditions, on the functions of the space $\overrightarrow{\widetilde{x}}_{h}$. So we introduce

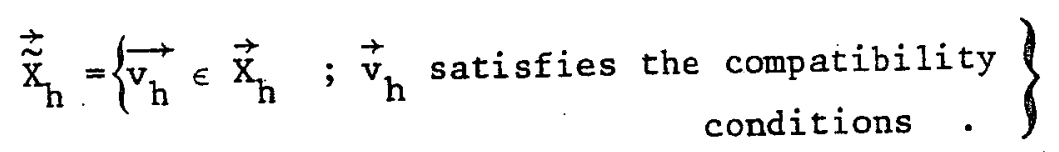

Next, in Theorem 3.2.1, we prove the existence of a "suitable"bijection $F_{h}$ between this space $\overrightarrow{\widetilde{x}}_{h}$ and the space $\vec{x}_{h}$ considered in section 2.1 .

Finally, in a third step, we shall take into account the boundary conditions and thus, we shall get the space $\vec{V}_{h}$.

Definition of the space $\stackrel{\vec{\pi}}{\vec{x}}_{h}$ :

To each partition $\mathscr{C}_{h}$ of the interval $\underset{\vec{I}}{\overrightarrow{\vec{I}}}($ see $(2.1 .1))$, we associate a product of finite element spaces $\overrightarrow{\widetilde{X}}_{h}=\tilde{\mathrm{X}}_{\mathrm{h}_{1}} \times \widetilde{\mathrm{X}}_{\mathrm{h}_{2}}$ defined as follows : In both spaces $\widetilde{\mathrm{x}}_{h \alpha}, \alpha=1,2$, the functions are independently defined segment by segment $\mathrm{K}_{\mathrm{i}} \in \mathscr{C}_{\mathrm{h}}$.

Space $\widetilde{\widetilde{x}}_{\mathrm{h}_{1}}:$

On a given $k_{i}$, the restriction $v_{h} / k_{i}$ of any function $v_{h}$ of $\widetilde{\mathbb{X}}_{h_{1}}$ is such that:

(i) $v_{h \mid K_{i}} \in \mathrm{P}_{1}\left(\mathrm{~K}_{i}\right)$;

(ii) $v_{h} / k_{i}$ is completely specified by its values at the extremities of $\mathrm{K}_{i}$. 
$-14-$

Space $\widetilde{\widetilde{X}}_{\mathrm{h}_{2}}$ :

On a given $K_{i}$, the restriction $v_{h / K_{i}}$ of any function $v_{h}$ of $\tilde{\tilde{X}}_{\mathrm{h}_{2}}$ is such that :

(i) $\mathrm{v}_{\mathrm{h} / \mathrm{K}_{i}} \in \mathrm{P}_{3}\left(\mathrm{~K}_{\mathrm{i}}\right)$;

(ii) $v_{h} / K_{i}$ is completely specified by its values and the values of its derivative at the extremitis of $\mathrm{K}_{i}$.

In the following, the space $\widetilde{\tilde{x}}_{\mathrm{h}_{1}}$ (resp. $\tilde{\mathrm{x}}_{\mathrm{h}_{2}}$ ) will contain the first (resp. third) component $\underset{\widetilde{\widetilde{v}}}{\tilde{\mathrm{v}}_{1}}$ (resp. $\widetilde{\widetilde{v}}_{\mathrm{h}_{3}}$ ) of the discrete displacement vector $\overrightarrow{\widetilde{v}}_{h}$ of the approximate $\operatorname{arch} a_{h}, i . e$. ,

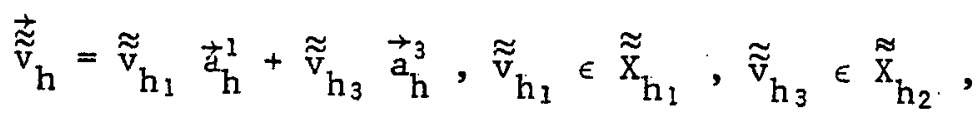

with possible discontinuities at mesh points of $a_{h}$.

Derivation of the compatibility conditions :

To derive the compatibility conditions, it is convenient to consider two adjacent elements $k^{+}$and $k^{-}$of $a_{h}$ which have a common extremity $\sigma$ (see Figure 3.2.1)


Fig. 3.2.1 
This common vertex $\sigma$ can be regarded as :

(i) a point of the arch $a$ : then, using the notations of section 1.1 , any displacement field $\vec{v} \in \vec{V}$ of the arch $a$ has the following components at point $\sigma$ or, more conveniently, at point $\Sigma$ (using the mapping $\vec{\phi}$ ). :

$$
\vec{v}(\Sigma)=v_{1}(\Sigma) \vec{a}^{1}(\Sigma)+v_{3}(\Sigma) \cdot \vec{a}^{3}(\Sigma)
$$

To the displacement field $\vec{v}$, we associate the usual rotation vector $\vec{\omega}$ (see $[17]$ ) which expression is given here by

$$
\begin{aligned}
& \vec{\omega}(\vec{v})=\omega^{i}(\vec{v}) \vec{a}_{i} \\
& \text { with }\left\{\begin{array}{l}
\omega^{2}(\vec{v})=-\frac{1}{\sqrt{a(\Sigma)}}\left(v_{3}^{\prime}(\Sigma)-\frac{1}{R(\Sigma)} v_{1}(\Sigma)\right) \\
\omega^{1}(\vec{v})=\omega^{3}(\vec{v})=0
\end{array}\right\}
\end{aligned}
$$

(ii) an extremity of the element $\mathrm{k}^{+}=\vec{\phi}_{\mathrm{h}}\left(\mathrm{k}^{+}\right)$.

To be more precise we use the superscript + to refer to parameters attached to $\mathrm{K}^{+}$. Thus, we write $\Sigma^{+}$instead of $\Sigma, \vec{a}_{h}^{i+}$ instead of $\vec{a}_{h}$, etc. Then any displacement field $\overrightarrow{\vec{v}}_{h} \in \overrightarrow{\widetilde{X}}_{h}$ of the approximate arch $a_{h}$ has the following, components at point $\sigma^{+}=\sigma$ (or, using $\vec{\phi}_{h}$, at point $\Sigma^{+}$) :

$$
\overrightarrow{\widetilde{v}}_{h}\left(\Sigma^{+}\right)=\widetilde{\widetilde{v}}_{h_{1}}\left(\Sigma^{+}\right){\overrightarrow{a_{h}^{1}}}_{h^{+}}^{+}+\widetilde{\widetilde{v}}_{h_{3}}\left(\Sigma^{+}\right) \vec{a}_{h}^{3^{+}}
$$

To the displacement field $\vec{\sim}_{\vec{\sigma}} \vec{\sigma}_{h}$, we associate the usual rotation vector $\overrightarrow{\widetilde{w}}_{h}$

$$
\begin{aligned}
& \overrightarrow{\widetilde{\sigma}}_{h}\left(\overrightarrow{\widetilde{v}}_{h}^{+}\right)=\widetilde{\widetilde{\omega}}_{h}^{i+} \vec{a}_{h i}^{+},
\end{aligned}
$$




(iii) an extremity of the element $k^{-}=\vec{\phi}_{h}\left(K^{-}\right)$

The results derive of those of (ii) by substituting the superscript - for the superscript + .

Now, we are able to get the compatibility conditions which consist in constraints imposed to the functions $\overrightarrow{\widetilde{v}}_{\mathrm{h}}$ of the space $\overrightarrow{\widetilde{x}}_{h}$, i.e., they correspond to relations that the degrees of freedom of the space $\vec{\pi}_{h}$ have to satisfy. We derive them by writting that :

$$
\begin{aligned}
& \text { (i) the displacement } \overrightarrow{\widetilde{v}}_{\mathrm{h}} \text { is continuous at the mesh points } \\
& \sigma=\vec{\phi}(\Sigma) \text {, i.e., } \\
& \left.\overrightarrow{\widetilde{v}}_{\mathrm{h}}\left(\Sigma^{+}\right)=\overrightarrow{\widetilde{v}}_{\mathrm{h}}\left(\Sigma^{-}\right), \forall \Sigma \text { mesh point of the }\right) \\
& \text { partition } \mathscr{C}_{\mathrm{h}} ;
\end{aligned}
$$

(ii) the nonzero component of the rotation vector $\overrightarrow{\widetilde{\omega}}_{h}\left(\overrightarrow{\widetilde{v}}_{h}\right)$ is continuous at the mesh points $\sigma=\bar{\phi}(\Sigma)$, i.e., with (3.2.7)

$$
\left.\begin{array}{l}
\frac{1}{\sqrt{a_{h}^{+}}} \approx_{h_{3}}^{\prime}\left(\Sigma^{+}\right)=\frac{1}{\sqrt{a_{h}}} \approx_{h_{3}}^{\prime}\left(\Sigma^{-}\right), \forall \Sigma \text { mesh point } \\
\text { of the partition } \mathscr{b}_{h} .
\end{array}\right\}
$$

Remark 3.2.1 : Let us observe that

(i) condition (3.2.9) is natural in the sense that the nonzero component is measured on the fixed basis vector $\vec{a}_{h_{2}}=\vec{a}_{h_{2}}=\vec{a}_{2}=\vec{e}_{3}$ (see $(3.1 .9) ;$

(ii) these compatibility conditions are written directly from the degrees of freedom of $\overrightarrow{\widetilde{x}}_{h}$. 
Let $\overrightarrow{\widetilde{x}}_{\mathrm{h}}$ be the following space

$\overrightarrow{\widetilde{X}}_{\mathrm{h}}=\left\{\begin{array}{l}\overrightarrow{\tilde{v}}_{\mathrm{h}} \in{\underset{\mathrm{x}}{\mathrm{h}}}_{\mathrm{Z}} ; \overrightarrow{\tilde{v}}_{\mathrm{h}} \text { satisfies the compatibility } \\ \text { conditions }(3.2 .8)(3.2 .9)\}\end{array}\right\}(3.2 .10)$

With the next theorem, the definition (3.2.10) will become clear.

Theorem 3.2.1. : There exists a "suitable" bijection $F_{h}$ between the spaces $\overrightarrow{\vec{x}}_{\mathrm{h}}$ and $\overrightarrow{\mathrm{x}}_{\mathrm{h}}$.

Proof :

(i) $\overrightarrow{\widetilde{v}}_{h} \in \overrightarrow{\widetilde{x}}_{h} \rightarrow \vec{v}_{h} \in \vec{x}_{h}$

By assumption $\overrightarrow{\widetilde{v}}_{\mathrm{h}} \in \overrightarrow{\widetilde{\mathrm{X}}}_{\mathrm{h}}$ so that $\overrightarrow{\mathrm{v}}_{\mathrm{h}}$ satisfies compatibility conditions $(3.2 .8)(3.2 .9)$. Hence to the function $\overrightarrow{\vec{v}}_{h} \in \overrightarrow{\widetilde{x}}_{h}$, we associate the function $\vec{v}_{h} \in \vec{X}_{h}$ whose degrees of freedom are uniquely determined by the relations

$$
\begin{aligned}
& \vec{v}_{h}(\Sigma)=\overrightarrow{\widetilde{v}}_{h}\left(\Sigma^{+}\right)=\overrightarrow{\widetilde{v}}_{h}\left(\Sigma^{-}\right), \forall \Sigma \text { mesh point of } \mathscr{\mathcal { G }}_{h} \\
& \mathrm{v}_{\mathrm{h}_{3}}^{\prime}(\Sigma)=\frac{\sqrt{\mathrm{a}(\Sigma)}}{\sqrt{\mathrm{a}_{\mathrm{h}}^{+}}} \tilde{\mathrm{v}}_{\mathrm{h}_{3}}^{\prime}\left(\Sigma^{+}\right)+\frac{1}{\mathrm{R}(\Sigma)} \mathrm{v}_{\mathrm{h}_{1}}(\Sigma) \\
& =\frac{\sqrt{a(\Sigma)}}{\sqrt{a_{h}^{-}}} \tilde{v}_{h_{3}}^{\prime}\left(\Sigma^{-}\right)+\frac{1}{R(\Sigma)} v_{h_{1}}(\Sigma),
\end{aligned}
$$

Converse1y, to any function $\vec{v}_{h}, \in \vec{x}_{h}$ the relations

$\overrightarrow{\tilde{v}}_{h}\left(\Sigma^{+}\right)=\overrightarrow{\widetilde{v}}_{h}\left(\Sigma^{-}\right)=\vec{v}_{h}(\Sigma)$ 


$$
\left.\begin{array}{l}
\frac{1}{\sqrt{a_{h}^{+}}} \tilde{v}_{h_{3}}^{\prime}\left(\Sigma^{+}\right)=\frac{1}{\sqrt{a_{h}^{-}}} \tilde{v}_{h_{3}}^{\prime}\left(\Sigma^{-}\right)=\frac{1}{\sqrt{a(\Sigma)}}\left(v_{h_{3}}^{\prime}(\Sigma)-\frac{1}{R(\Sigma)} v_{h_{1}}(\Sigma)\right), \\
\forall \Sigma \text { mesh point of the partition } \ddot{b}_{h}
\end{array}\right\}
$$

associate one and only one function $\overrightarrow{\widetilde{v}}_{h} \in \overrightarrow{\widetilde{x}}_{h}$

Let us note that, in general, we do not have

$\overrightarrow{\widetilde{x}}_{h} \subset H^{2}(I) \times H^{2}(I)$

but only

$$
\overrightarrow{\tilde{X}}_{h} \subset \prod_{i=1}^{M_{h}}\left(H^{1}\left(K_{i}\right) \times H^{2}\left(K_{i}\right)\right)
$$

Consequertily, the norm of the space $\overrightarrow{\widetilde{x}}_{h}$ is defined by

$$
\left\|\overrightarrow{\tilde{v}}_{h}\right\|_{h}=\sum_{i=1}^{M_{h}}\left(\left\|\tilde{v}_{h_{1}}\right\|_{1}^{2}, k_{i}+\left\|\tilde{v}_{h_{3}}\right\|_{2, K_{i}}^{2}\right)^{1 / 2}
$$

Definition of the space $\overrightarrow{\widetilde{V}}_{\mathrm{h}}$ :

In order to formulate the discrete problem, we define the subspace $\overrightarrow{\vec{V}}_{h}$ of the space $\overrightarrow{\widetilde{X}}_{h}$ :

$$
\left.\begin{array}{l}
\overrightarrow{\tilde{v}}_{h}=\left\{\overrightarrow{\tilde{v}}_{h}=\left(\tilde{v}_{h_{1}}, \tilde{v}_{h_{3}}\right) \in \overrightarrow{\tilde{x}}_{h} ;\right. \\
\left.\tilde{v}_{h_{1}}(0)=\tilde{v}_{h_{3}}(0)=\tilde{v}_{h_{3}}^{\prime}(0)=\tilde{v}_{h_{1}}(L)=\tilde{v}_{h_{3}}(L)=\tilde{v}_{h_{3}}^{\prime}(L)=0\right\}
\end{array}\right\}(3.2 \cdot 16)
$$

It $\underset{t}{\vec{*}}$ clear that the mapping $F_{h}$ define a bijection between the spaces $\overrightarrow{\tilde{V}}_{h}$ and $\vec{V}_{h}$ where, from $(2.1 .4)(2.1 .5)$,

$$
\left.\begin{array}{l}
\vec{v}_{h}=\left\{\vec{v}_{h}=\left(v_{h_{1}}, v_{h_{3}}\right) \in \vec{x}_{h} ;\right. \\
\left.v_{h_{1}}(0)=v_{h_{3}}(0)=v_{h_{3}}^{\prime}(0)=v_{h_{1}}(L)=v_{h_{3}}(L)=v_{h_{3}}^{\prime}(L)=0\right\}
\end{array}\right\}
$$




\section{3 - The discrete problem :}

According to the expression (1.2.3) of the bilinear form $a(.,$.$) , a natural candidate for the approximated bilinear form is$ given by piecing together all the elementary energies, i.e., for the associated bilinear form :

$$
\begin{aligned}
& \tilde{a}_{h}\left(\overrightarrow{\tilde{u}}_{h}, \overrightarrow{\tilde{v}}_{h}\right)=\sum_{i=1}^{M_{h}} \int_{K_{i}} \frac{E e}{1-v^{2}}\left\{\tilde{\gamma}_{h_{l}}{ }^{2}\left(\overrightarrow{\tilde{u}}_{h}\right) \tilde{\gamma}_{h_{l}}{ }^{1}\left(\overrightarrow{\tilde{v}}_{h}\right)+\right.
\end{aligned}
$$

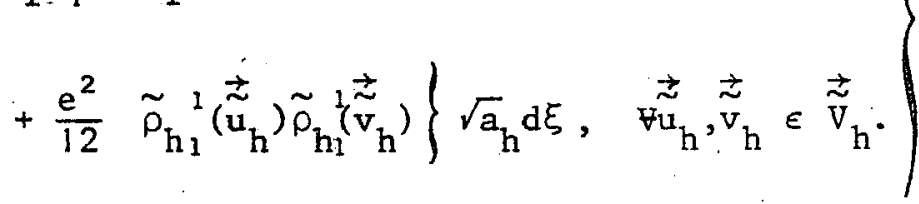

Over this expression, one can check that the approximated strain energy $\frac{1}{2} \tilde{a}_{h}\left(\overrightarrow{\widetilde{v}}_{h}, \overrightarrow{\widetilde{v}}_{h}\right)$ of the arch is the sum of uncoupled elementary energies of straight beams in extension, on the one hand, and in bending, on the other hand. For clarity, we record that.

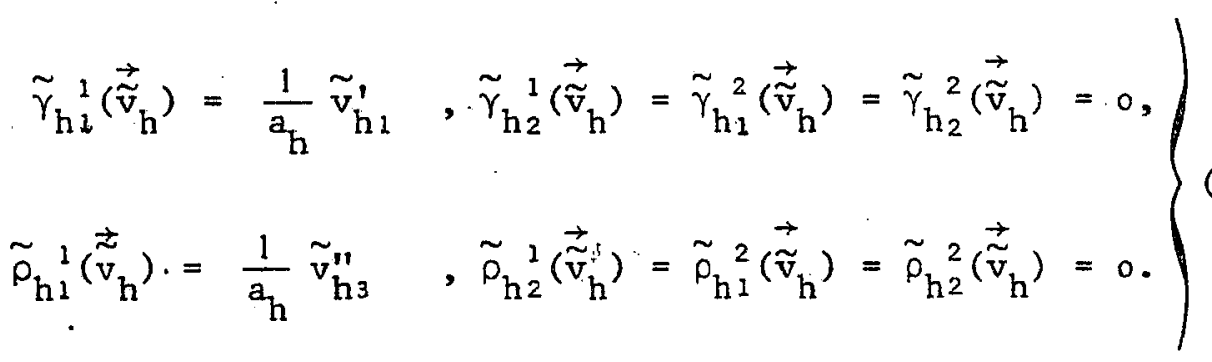

The integrals over the segments $k_{i}$ which appear in (3.3.1) are seldom computed exactly in practice, but rather numerical integration schemes are used. Thus, let us consider a numerical integration scheme over the reference segment $\hat{\mathrm{K}}=] 0,1[$ :

$$
\int_{\mathrm{K}} \hat{\phi}(\hat{\mathrm{x}}) \mathrm{d} \hat{\mathrm{x}} \sim \sum_{\ell=1}^{\mathrm{L}} \hat{\omega}_{\ell} \hat{\phi}\left(\hat{\mathrm{b}}_{\ell}\right) .
$$

The segment $k_{i}$ defined in $(2.1 .1)$ is associated to the segment $\hat{K}$ through the bijection 


$$
\mathrm{F}_{\mathrm{K}_{i}}: \hat{\mathrm{x}} \in \hat{\mathrm{K}} \rightarrow \mathrm{F}_{\mathrm{K}_{i}}(\hat{\mathrm{x}})=\left(\xi_{\hat{i}}-\xi_{i-1}\right) \hat{\mathrm{x}}+\xi_{i-1}=\xi \text {. }
$$

Then, using the usual correspondence between $\phi$ and $\hat{\phi}$ through the mapping $\mathrm{F}_{\mathrm{K}_{\mathrm{i}}}$, we obtain

$$
\int_{K_{i}} \phi(\xi) d \xi=\left(\xi_{i}-\xi_{i-1}\right) \int_{\hat{K}} \hat{\phi}(\hat{x}) \mathrm{d} \hat{x} .
$$

The relations (3.3.3) to (3.3.5) induce the following numerical integration scheme over the segment $\mathrm{K}_{i}, i=1, \ldots, \mathrm{M}_{\mathrm{h}}$ :

$$
\int_{\mathrm{K}_{\mathrm{i}}} \phi(\xi) \mathrm{d} \xi \sim \sum_{\ell=1}^{\mathrm{L}} \omega_{\ell, \mathrm{K}_{i}} \phi\left(\mathrm{b}_{\ell, \mathrm{K}_{\mathrm{i}}}\right)
$$

where

$$
\omega_{\ell, k_{i}}=\left(\xi_{i}-\xi_{i-1}\right) \hat{\omega}_{\ell}, b_{\ell, K_{i}}=F_{K_{i}}\left(\hat{b}_{\ell}\right), 1 \leq \ell \leq L . \quad(3.3 .7)
$$

Moreover, we define the error functionals

$$
\begin{aligned}
& \hat{E}(\hat{\phi})=\int_{\hat{K}} \hat{\phi}(\hat{\mathbf{x}}) \mathrm{d} \hat{\mathrm{x}}-\sum_{\ell=1}^{\mathrm{L}} \hat{\omega}_{\ell} \hat{\phi}\left(\hat{\mathrm{b}}_{\ell}\right), \\
& \mathrm{E}_{\mathrm{K}_{\mathrm{i}}}(\phi)=\int_{\mathrm{K}_{i}} \phi(\xi) \mathrm{d} \xi-\sum_{\ell=1}^{L} \omega_{\ell, \mathrm{K}_{i}} \phi\left(\mathrm{b}_{\ell, \mathrm{K}_{i}}\right),
\end{aligned}
$$

so that

$$
E_{K_{i}}(\phi)=\left(\xi_{i}-\xi_{i-1}\right) \hat{E}(\hat{\phi})
$$


Thus, from the expression (3.3.1), we define a new approximated bilinear form

$$
\begin{aligned}
& a_{h}^{*}\left(\overrightarrow{\tilde{u}}_{h}, \overrightarrow{\widetilde{v}}_{h}\right)=\sum_{i=1}^{M_{h}} \sqrt{a_{h}}\left[\sum_{\ell_{1}=1}^{L_{1}} \omega_{\ell_{1, K}}\left\{\frac{E e}{1-v^{2}} \tilde{\gamma}_{h 1}^{1}\left(\overrightarrow{\tilde{u}}_{h}\right) \tilde{\gamma}_{h 1}^{1}\left(\overrightarrow{\tilde{v}}_{h}\right)\right\}\left\{b_{\ell}, K_{i}\right\}\right. \\
& \left.+\sum_{\ell_{2}=1}^{L_{2}} \omega_{\ell_{2, K_{i}}}\left\{\frac{E e^{3}}{12\left(1-v^{2}\right)} \tilde{\rho}_{h_{1}}{ }^{1}\left(\overrightarrow{\tilde{u}}_{h}\right) \tilde{\rho}_{h_{1}}{ }^{1}\left(\vec{v}_{h}\right)\right\}\left\{b_{\ell_{2, K_{i}}}\right\}\right]
\end{aligned}
$$

In the last expression, we have used two differents numerical integration schemes in order to take into account the uncoupling between the membrane terms, i.e., $\tilde{\gamma}_{h_{1}{ }^{1}}$, and the bending terms, i.e., $\tilde{\rho}_{h_{1}}{ }^{1}$, each of them being approximated in two different finite element spaces.

Next, to the linear form $(1.2 .4)$, we associate the approximated liriear form $f_{h}$, i.e.,

$$
\begin{aligned}
& f_{h}\left(\vec{v}_{h}\right)=\frac{1}{2} \sum_{i=1}^{M_{h}}\left(\xi_{i}-\xi_{i-1}\right)\left[\left(\vec{p} \cdot \vec{v}_{h} \sqrt{a}\right)\left(\xi_{i-1}\right)+\left(\vec{p}_{v_{h}} \sqrt{a}\right)\left(\xi_{i}\right)\right] \\
& \forall \vec{v}_{h} \in \vec{v}_{h} .
\end{aligned}
$$

By using the bijection $\mathrm{F}_{\mathrm{h}}: \overrightarrow{\widetilde{v}}_{\mathrm{h}} \in \overrightarrow{\widetilde{v}}_{\mathrm{h}} \rightarrow \overrightarrow{\mathrm{v}}_{\mathrm{h}} \in \overrightarrow{\mathrm{v}}_{\mathrm{h}}$ defined in Theorem 3.2.1, we can define the following approximated forms

$$
b_{h}\left(\vec{u}_{h}, \vec{v}_{h}\right)=a_{h}^{*}\left(\overrightarrow{\tilde{u}}_{h}, \overrightarrow{\tilde{v}}_{h}\right)
$$

for all $\vec{u}_{\mathrm{h}}, \vec{v}_{\mathrm{h}} \in \overrightarrow{\mathrm{v}}_{\mathrm{h}}$ and all $\overrightarrow{\mathrm{u}}_{\mathrm{h}}, \overrightarrow{\widetilde{v}}_{\mathrm{h}} \in \overrightarrow{\widetilde{v}}_{\mathrm{h}}$ associated through the bijection $\mathrm{F}_{\mathrm{h}}$.

Then, the discrete problem using straight beam elements can be stated as follows : Find an element $\vec{u}_{h} \in \vec{v}_{h}$ such that 


$$
b_{h}\left(\vec{u}_{h}, \vec{v}_{h}\right)=E_{h}\left(\vec{v}_{h}\right), \vec{v}_{h} \in \vec{v}_{h}
$$

Remark 3.3.1 : By analogy to (3.3.1), we could define an approximated linear form as a sum of integrals over the intervals $R_{i}$. However, we have defined the right-hand side of the discrete problem (3.3.14) as in engineering literature, where it is considered that the applied forces are approximated by a sum of concentrated forces. Indeed, note that (3.3.12) can be rewritten, for all $\vec{v}_{h} \in \vec{v}_{h}$

$$
\begin{aligned}
f_{h}\left(\vec{v}_{h}\right) & =\frac{1}{2}\left(\xi_{1}-\xi_{0}\right)\left[\left(\vec{p} \cdot \vec{v}_{h} \sqrt{a}\right)\left(\xi_{0}\right)\right] \\
& +\frac{1}{2} \sum_{i=1}^{M_{h}-1}\left(\xi_{i+1}-\xi_{i-1}\right)\left[\left(\vec{p} \cdot \vec{v}_{h} \sqrt{a}\right)\left(\xi_{i}\right)\right] \\
& +\frac{1}{2}\left(\xi_{M_{h}}-\xi_{M_{h}-1}\right)\left[\left(\vec{p} \cdot \vec{v}_{h} \sqrt{a}\right)\left(\xi_{M_{h}}\right)\right] .
\end{aligned}
$$

We shall see that this simplification is theoretically justified since it does not decrease the order of convergence. Moreover, with respect to this order of convergence, we could use more simple numerical integration schemes, as quoted in Remark 4.3.1.

Remark 3.3.2 : In this section we have defined the discrete problem by using the finite element space $\overrightarrow{\tilde{V}}_{h}$ - see $(3.3 .1)$ and (3.3.11) for example. From section 3.2, it is obvious that the inclusion

$$
\overrightarrow{\tilde{\nabla}}_{\mathrm{h}} \subset \mathrm{H}^{2}(I) \times \mathrm{H}^{2}(I)
$$

no longer holds. We only have

$$
\overrightarrow{\tilde{v}_{h}} \subset \prod_{i=1}^{M_{h}}\left[H^{1}\left(K_{i}\right) \times H^{2}\left(K_{i}\right)\right] \text {. }
$$


This is why in the Introduction we have qualified this approach a "pseudo-conforming" approximation of the components of the displacement.

4 - CONVERGENCE AND ERROR ESTIMATES

In this paragraph, we shall be interested in the following problems :

(i) show that the problem (3.3.14) has a unique solution $\vec{u}_{h} \in \vec{v}_{h}$ (ii) show the error estimate

$$
\left\|\overrightarrow{\mathrm{u}}-\vec{u}_{\mathrm{h}}\right\|=0(\mathrm{~h})
$$

(i.e., the same order as in (2.2.2)), where $\vec{u}$ denotes the solution of the continuous problem (1.2.5).

From now on, we assume that the mapping $\vec{\phi}$ defined in $(1.1 .1)$ is sufficiently regular for our following purposes.

In order to solve the problem (ii), we shall first give an abstract error estimate.

\section{1 - Abstract error estimate :}

Theorem 4.1.1.: Let us consider a family of discrete problems (3.3.14) for which the bilinear forms are $\overrightarrow{\mathrm{v}}_{\mathrm{h}}$ - elliptic, uniformly whith respect to $h$, in the sense that there exists a constant $\alpha:>0$, independent of $h$, such that

$$
\alpha\left\|\vec{v}_{h}\right\|^{2} \leq b_{h}\left(\vec{v}_{h}, \vec{v}_{h}\right) \quad, \quad \forall \vec{v}_{h} \in \vec{v}_{h}
$$

Then, there exists a constant $C$, independent of $h$, such that 


$$
\begin{aligned}
& \left\|\vec{u}-\vec{u}_{h}\right\| \leq C\left\{\underset { \vec { v } _ { h } \in \vec { v } _ { h } } { i n f } \left\{\left\|\vec{u}-\vec{v}_{h}\right\|+\right.\right. \\
& \left.+\underset{\substack{\sup _{h} \in \vec{v}_{h}}}{ } \frac{\left|a\left(\vec{v}_{h}, \vec{w}_{h}\right)-b_{h}\left(\vec{v}_{h}, \vec{w}_{h}\right)\right|}{\left\|\vec{w}_{h}\right\|}\right\}+ \\
& \left.+\sup _{\vec{w}_{h} \in v_{h}} \frac{\left|f\left(\vec{w}_{h}\right)-f_{h}\left(\vec{w}_{h}\right)\right|}{\left\|\vec{w}_{h}\right\|}\right\}
\end{aligned}
$$

Proof : The assumption of $\vec{V}_{h}$ - ellipticity involves the existence and the uniqueness of a solution $\vec{u}_{h}$ for the discrete problem (3.3.14). Then, let $\vec{v}_{h}$ be any element of the space $\vec{v}_{h}$. We are able to write:

$$
\begin{aligned}
\alpha\left\|\vec{u}_{h}-\vec{v}_{h}\right\|^{2} & \leq b_{h}\left(\vec{u}_{h}-\vec{v}_{h}, \vec{u}_{h}-\vec{v}_{h}\right) \\
& =a\left(\vec{u}-\vec{v}_{h}, \vec{u}_{h}-\vec{v}_{h}\right)+\left[a\left(\vec{v}_{h}, \vec{u}_{h}-\vec{v}_{h}\right)-b_{h}\left(\vec{v}_{h}, \vec{u}_{h}-\vec{v}_{h}\right)\right] \\
& +\left[f_{h}\left(\vec{u}_{h}-\vec{v}_{h}\right)-f\left(\vec{u}_{h}-\vec{v}_{h}\right)\right]
\end{aligned}
$$

so that the continuity of the bilinear form a $(.,$.$) involves:$

$$
\begin{gathered}
\alpha\left\|\vec{u}_{h}-\vec{v}_{h}\right\| \leq M\left\|\vec{u}-\vec{v}_{h}\right\|+\frac{\left|a\left(\vec{v}_{h}, \vec{u}_{h}-\vec{v}_{h}\right)-b_{h}\left(\vec{v}_{h}, \vec{u}_{h}-\vec{v}_{h}\right)\right|}{\left\|\vec{u}_{h}-\vec{v}_{h}\right\|}+ \\
+\frac{\left|f\left(\vec{u}_{h}-\vec{v}_{h}\right)-f_{h}\left(\vec{u}_{h}-\vec{v}_{h}\right)\right|}{\left\|\vec{u}_{h}-\vec{v}_{h}\right\|},
\end{gathered}
$$

and thus

$$
\begin{aligned}
\alpha\left\|\vec{u}_{h}-\vec{v}_{h}\right\| & \leq M\left\|\vec{u}-\vec{v}_{h}\right\|+\underset{\vec{w}_{h} \in \vec{V}_{h}}{\sup _{h}} \frac{\left|a\left(\vec{v}_{h}, \vec{w}_{h}\right)-b_{h}\left(\vec{v}_{h}, \vec{w}_{h}\right)\right|}{\left\|\vec{w}_{h}\right\|}+ \\
& +\underset{\substack{\vec{w}_{h} \in \vec{V}_{h}}}{ } \frac{\left|f\left(\vec{w}_{h}\right)-f_{h}\left(\vec{w}_{h}\right)\right|}{\left\|\vec{w}_{h}\right\|} .
\end{aligned}
$$


Combining this inequality with the triangular inequality

$$
\left\|\vec{u}-\vec{u}_{h}\right\| \leq\left\|\vec{u}-\vec{v}_{h}\right\|+\left\|\vec{v}_{h}-\vec{u}_{h}\right\|
$$

and taking the minimum with respect to $\vec{v}_{h} \in \vec{v}_{h}$, we get the inequality $(4,1.2)$.

Thus, in addition to the usual approximation theory term $\underset{\vec{v}_{h} \in \vec{v}_{h}}{\inf }\left\|\vec{u}-\vec{v}_{h}\right\| \vec{v}$, we find two additional terms which measure the consistency between the bilinear forms $a(.,$.$) and b_{h}(.,$.$) on$ the one hand, and between the linear forms $f($.$) and g_{h}($.$) on the$ other hand.

4.2 - Estimate of the consistency error $\left|a\left(\vec{v}_{h}, \vec{w}_{h}\right)-b_{h}\left(\vec{v}_{h}, \vec{w}_{h}\right)\right|:$

Let us consider any functions $\vec{v}_{h}, \vec{w}_{h} \in \vec{x}_{h}$ and let us denote $\overrightarrow{\tilde{v}}_{h}, \overrightarrow{\tilde{w}}_{h} \in \overrightarrow{\vec{x}}_{h}$ the corresponding functions through the bijection $F_{h}$ defined in Theorem 3.2.1. Using definitions (3.3.1) (3.3.13) we have

$$
\left.\begin{array}{c}
a\left(\vec{v}_{h}, \vec{w}_{h}\right)-b_{h}\left(\vec{v}_{h}, \vec{w}_{h}\right)=a\left(\vec{v}_{h}, \vec{w}_{h}\right)-\tilde{a}_{h}\left(\overrightarrow{\tilde{v}}_{h}, \overrightarrow{\tilde{w}}_{h}\right) \\
+\tilde{a}_{h}\left(\overrightarrow{\tilde{v}}_{h}, \overrightarrow{\tilde{w}}_{h}\right)-\vec{a}_{h}^{*}\left(\overrightarrow{\tilde{v}}_{h}, \overrightarrow{\tilde{w}}_{h}\right) .
\end{array}\right\}
$$

In order to estimate the expression $\left|a\left(\vec{v}_{h}, \vec{w}_{h}\right)-\tilde{a}_{h}\left(\overrightarrow{\tilde{v}}_{h}, \overrightarrow{\tilde{w}}_{h}\right)\right|$ - see Theorem 4.2.3-we begin by proving preliminary Theorems 4.2.1 and 4.2.2. Next, in Theorem 4.2.4 we give an estimate for the difference $\left|\tilde{a}_{h}\left(\overrightarrow{\tilde{v}}_{h}, \overrightarrow{\tilde{w}}_{h}\right)-a_{h}{ }_{h}^{*}\left(\overrightarrow{\tilde{v}}_{h}, \overrightarrow{\tilde{w}}_{h}\right)\right|$ so that, by assembling it with the result of Theorem 4.2.3, we obtain, in Theorem 4.2.5, the estimate of the consistency error $\left|a\left(\vec{v}_{h}, \vec{w}_{h}\right)-b_{h}\left(\vec{v}_{h}, \vec{w}_{h}\right)\right|$.

Theorem 4.2.1.: There exists a constant $C$, independent of $h$, such that for any $\vec{v}_{h}=\left(v_{h_{1}}, v_{h_{3}}\right) \in \overrightarrow{\mathrm{x}}_{h}$ and any $\overrightarrow{\widetilde{v}}_{h}=\left(\tilde{v}_{h_{1}}, \tilde{v}_{h_{3}}\right) \in \overrightarrow{\widetilde{x}}_{h}$ in correspondence through the bijection $F_{h}$ defined in Theorem 3.2.1, we have for any $i, 1 \leq i \leq M_{h}$ : 


$$
\left|\tilde{\gamma}_{h_{1}}^{1}\left(\vec{v}_{h}\right)-\gamma_{1}^{3}\left(\vec{v}_{h}\right)\right|_{0, K_{i}} \leq C h\left\{\left\|v_{h i}\right\|_{1, k_{i}}^{2}+\left\|v_{h}\right\|_{2,}^{2}, k_{i}\right\}^{1 / 2}
$$

Proof (in three steps):

We record that from $(1.1 .17)$ and $(3.3 .2)$, we have

$$
\gamma_{1}^{1}\left(\vec{v}_{h}\right)=\frac{1}{\sqrt{a}}\left\{\left(\frac{v_{h_{1}}}{\sqrt{a}}\right)^{\prime}+\frac{\sqrt{a}}{R} v_{h_{3}}\right\}, \tilde{\gamma}_{h_{1}}^{1}\left(\vec{v}_{h}\right)=\frac{1}{a_{h}} \tilde{v}_{h_{1}}^{\prime} .
$$

Step 1 : An expression of $\tilde{v}_{h_{1}}^{\prime}(\xi)$ using $\vec{v}_{h}=F_{h}\left(\overrightarrow{\tilde{v}}_{h}\right)$.

Let us consider the restriction $\left.\vec{\sim}_{\mathrm{h}}\right|_{\mathrm{K}_{i}}$ to any element

$\left.\mathrm{K}_{i}=\right] \xi_{i-1}, \xi_{j}[$ of the partition of $I$. For simplicity, we still denote $\overrightarrow{\vec{v}}_{h}$ this restriction. By definition

$$
\begin{aligned}
& \overrightarrow{\widetilde{v}}_{h}=\tilde{v}_{h_{1}} \vec{a}_{h}^{1}+\tilde{v}_{h_{3}} \vec{a}_{h}^{3}, \tilde{v}_{h_{1}} \epsilon \tilde{v}_{h_{1}}, \tilde{v}_{h_{3}} \in \tilde{v}_{h_{2}} . \\
& \text { Thus, } \tilde{v}_{h_{1}} \in P_{1}\left(k_{i}\right) \text { so that, for any } \xi \in\left[\xi_{i-1}, \xi_{i}\right] \\
& \tilde{v}_{h_{1}}^{\prime}(\xi)=\frac{\tilde{v}_{h_{1}}\left(\xi_{i}\right)-\tilde{v}_{h_{1}}\left(\xi_{i-1}\right)}{\xi_{i}-\xi_{i-1}} .
\end{aligned}
$$

$$
\left.\begin{array}{l}
\text { Moreover, from the relations }(3.2 .11) \text {, we obtain } \vec{\sim}_{h}\left(\xi_{i}\right)=\vec{v}_{h}\left(\xi_{i}\right) \text {. } \\
\text { Since } \vec{v}_{h}(\xi)=v_{h_{1}}(\xi) \vec{a}^{\frac{1}{a}}(\xi)+v_{h_{3}}(\xi) \cdot \vec{a}^{3}(\xi) \text {, we obtain } \\
\left.\tilde{v}_{h_{1}}\left(\xi_{i}\right)=\left(\vec{a}^{1}\left(\xi_{i}\right) \cdot \vec{a}_{h_{1}}\right) v_{h_{1}}\left(\xi_{i}\right)+\left(\vec{a}^{3}\left(\xi_{i}\right) \cdot \vec{a}_{h_{1}}\right) v_{h_{3}}\left(\xi_{i}\right),\right\} \\
i=1, \ldots, M_{h} .
\end{array}\right\}
$$

Let us denote

$$
d_{h k}^{j}(\xi)=\vec{a}^{j}(\xi) \cdot \vec{a}_{h k}
$$


Then, the relations $(4.2 .4)(4.2 .5)$ involve for any $\xi \in \mathrm{K}_{i}$

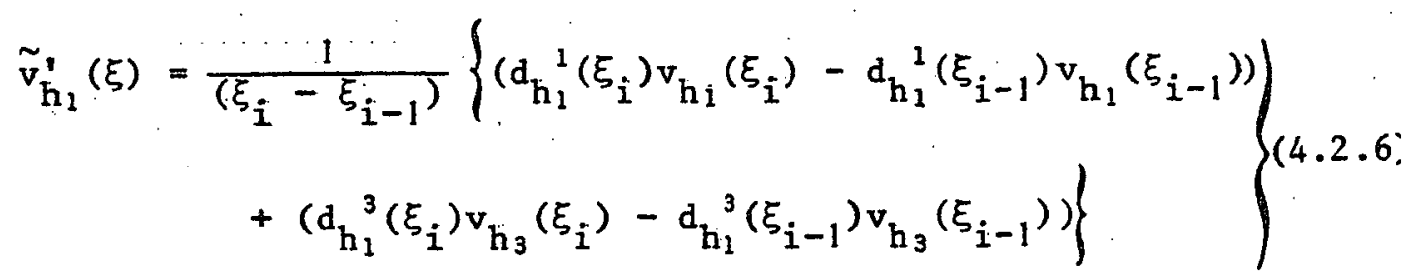

Step 2 : Finite expansion of $\widetilde{v}_{h_{1}}^{\prime}(\xi)$.

For simplicity, we denote $\vec{\phi}(\xi)$ for $\vec{\phi}(\xi, 0)$. Then, the regularity of $\vec{\phi}$ permits to write (see $(1.1 .4))$ :

$$
\vec{a}_{1}(\Sigma)=\vec{a}_{1}(\xi)+(\Sigma-\xi) \vec{\phi}^{\prime \prime}(\xi)+o\left(h^{2}\right), \quad \Sigma=\xi_{i} \text { or } \xi_{i-1}
$$

Here and subsequently, the notation $g(\xi)=O\left(h^{k}\right)$ means that there exists a constant $C$ independent of $h$ such that

$$
\begin{aligned}
& \sup _{\xi \in I_{i}}|g(\xi)| \leq \mathrm{Ch}^{\mathrm{k}} \\
& a(\Sigma)=a(\xi)+2(\Sigma-\xi)\left(\vec{\phi}^{\prime}(\xi) \cdot \vec{\phi}^{\prime \prime}(\xi)\right)+o\left(h^{2}\right), \\
& \frac{1}{a(\Sigma)}=\frac{1}{a(\xi)}\left\{1-2 \frac{(\Sigma-\xi)}{a(\xi)}\left(\vec{\phi}^{\prime}(\xi) \cdot \vec{\phi}^{\prime \prime}(\xi)\right)+o\left(h^{2}\right)\right\} . \\
& \text { From }(1.1 .10),(4.2 .7) \text { and }(4.2 .9) \text {, we deduce } \\
& \vec{a}^{1}(\Sigma)=\frac{1}{a(\xi)}\left\{\vec{a}_{1}(\xi)+(\Sigma-\xi) \vec{\phi}^{\prime \prime}(\xi)-\right. \\
& \left.-2 \frac{(\Sigma-\xi)}{a(\xi)}\left(\vec{\phi}^{\prime}(\xi) \cdot \vec{\phi}^{\prime \prime}(\xi)\right) \vec{a}_{1}(\xi)+o\left(h^{2}\right)\right\}
\end{aligned}
$$




\section{Likewise}

$$
\vec{a}_{3}(\Sigma)=\vec{a}_{3}(\xi)+(\Sigma-\xi) \vec{a}_{3}^{\prime}(\xi)+0\left(h^{2}\right)
$$

Owing to the regularity of $\vec{\phi}$, we can write

$$
\vec{\phi}(\Sigma)=\vec{\phi}(\xi)+(\Sigma-\xi) \vec{\phi}^{\prime}(\xi)+\frac{1}{2}(\Sigma-\xi)^{2} \bar{\phi}^{\prime \prime}(\xi)+O\left(\mathrm{~h}^{3}\right),(4.2 .12)
$$

so that we obtain from $(1.1 .7)$ and $(3.1 .8)$

$$
\vec{a}_{h_{1}}=\vec{a}_{1}(\xi)+\frac{1}{2}\left(\xi_{i}+\xi_{i-1}-2 \xi\right) \vec{\phi}(\xi)+o\left(h^{2}\right) .
$$

Combining the above expansion with $(4.2 .10)$ and $(4.2 .11)$ we deduce :

$$
\begin{aligned}
& \left.\begin{array}{rl}
d_{h i}^{1}(\Sigma)= & + \\
& +\frac{1}{2 a(\xi)}\left\{\xi_{i}+\xi_{i-1}-2 \Sigma\right\}\left(\bar{\phi}^{\prime}(\xi) \cdot \vec{\phi}^{\prime \prime}(\xi)\right)+o\left(h^{2}\right)
\end{array}\right\} \\
& \mathrm{d}_{\mathrm{h}_{1}}{ }^{3}(\Sigma)=(\Sigma-\xi)\left(\vec{a}_{3}^{\prime}(\xi) \cdot \vec{\Phi}^{\prime}(\xi)\right) \\
& \left.+\frac{1}{2}\left(\xi_{i}+\xi_{i-1}-2 \xi\right)\left(\vec{a}_{3}(\xi) \cdot \phi^{\prime \prime}(\xi)\right)+O\left(h^{2}\right) \cdot\right\} \\
& \text { From (1.1.2), (1.1.11) and (1.1.13), we obtain } \\
& \vec{a}_{3}^{\prime}(\xi) \cdot \vec{\phi}^{\prime}(\xi)=\vec{a}_{3}^{\prime}(\xi) \cdot \vec{a}_{1}(\xi)=-\vec{a}_{3}(\xi) \cdot \vec{a}_{1}^{\prime}(\xi)=\frac{a(\xi)}{R(\xi)} \\
& \text { so that relation }(4.2 .15) \text { becomes } \\
& d_{h_{1}}^{3}(\Sigma)=\left(2 \Sigma-\xi_{i}-\xi_{i-1}\right) \frac{a(\xi)}{2 R(\xi)}+O\left(h^{2}\right)
\end{aligned}
$$


Then, since $v_{h_{1}} \in P_{1}\left(R_{i}\right)$ and $v_{h_{3}} \in P_{3}\left(R_{i}\right)$, there exists $n \in\left[\xi_{i-1}, \xi_{i}\right]$ such that

$$
\left.\begin{array}{l}
v_{h_{1}}(\Sigma)=v_{h_{1}}(\xi)+(\Sigma-\xi) v_{h_{1}}^{\prime}, \\
v_{h_{3}}(\Sigma)=v_{h_{3}}(\xi)+(\Sigma-\xi) v_{h_{3}}^{\prime}(\eta) \cdot
\end{array}\right\}
$$

Combining the finite expansions $(4.2 .14)(4.2 .17)(4.2 .18)$ with relations $(4.2 .6)$

$$
\left.\begin{array}{rl}
\tilde{v}_{h_{1}}^{\prime}(\xi) \geqslant & v_{h_{1}}^{\prime}(\xi)-\frac{1}{a(\xi)}\left(\dot{\phi}^{\prime}(\xi) \cdot \vec{\phi}^{\prime \prime}(\xi)\right) v_{h_{1}}(\xi)+ \\
& +\frac{a(\xi)}{R(\xi)} v_{h_{3}}(\xi)+\left(\dot{v}_{h_{1}}^{\prime}(\xi)+v_{h_{1}}^{\prime}+v_{h_{3}}(\xi)+\right. \\
& \left.+v_{h_{3}}^{\prime}(n)\right) o(h) .
\end{array}\right\}(4.2 .19)
$$

Step 3 : Obtention of estimate $(4.2 .2)$

Relations (3.1.8) and (3.1.9) involve 
$-30-$

$$
\left.\begin{array}{c}
a_{h}=\frac{1}{\left(\xi_{i}-\xi_{i-1}\right)^{2}}\left\{\left(\phi^{1}\left(\xi_{i}\right)-\phi^{1}\left(\xi_{i-1}\right)\right)^{2}+\right. \\
\left.+\left(\phi^{2}\left(\xi_{i}\right)-\phi^{2}\left(\xi_{i-1}\right)\right)^{2}\right\},
\end{array}\right\}
$$

so that we deduce with $(4.2 .12)$ and $(1.1 .3)$

$$
\sqrt{a_{h}}=\sqrt{a(\xi)}+o(h)
$$

Then, from $(4.2 .19)$ and $(4.2 .21)$, we obtain for any $\xi \in \overline{\mathrm{k}}_{i}$,

$$
\begin{aligned}
\frac{\tilde{v}_{h_{1}}^{\prime}(\xi)}{\sqrt{a_{h}}}= & \left(\frac{v_{h_{1}}(\xi)}{\sqrt{a(\xi)}}\right)^{\prime}+\frac{\sqrt{a(\xi)}}{R(\xi)} v_{h_{3}}(\xi)+ \\
& +\left(v_{h_{1}}(\xi)+v_{h_{1}}^{\prime}+v_{h_{3}}(\xi)+v_{h_{3}}^{\prime}(\eta)\right) O(h)
\end{aligned}
$$

and thus, with $(4.2 .3)$,

$$
\left.\begin{array}{rl}
\tilde{\gamma}_{h_{1}}^{1}\left(\overrightarrow{\tilde{v}}_{h}\right)= & \gamma_{1}^{1}\left(\vec{v}_{h}\right)+\left(v_{h_{1}}(\xi)+v_{h_{1}}^{\prime}+v_{h_{3}}(\xi)+\right. \\
& \left.+v_{h_{3}}^{\prime}(\eta)\right) o(h)
\end{array}\right\}(4.2 .22)
$$

From (4.2.22), we derive for any element $k_{i} \in \mathscr{V}_{h}$ :

$$
\left.\begin{array}{l}
\left|\tilde{\gamma}_{h_{1}}{ }^{1}\left(\overrightarrow{\tilde{v}}_{\mathrm{h}}\right)-\gamma_{1}^{1}\left(\vec{v}_{\mathrm{h}}\right)\right|_{0, \mathrm{~K}_{i}} \leq \\
\leq \underset{\mathrm{p}=0}{\mathrm{Ch}}{ }^{3 / 2} \sum^{1}\left\{\left|\mathrm{v}_{\mathrm{h}_{1}}\right|_{\mathrm{p}, \infty, \mathrm{K}_{i}}^{2}+\left|\mathrm{v}_{\mathrm{h}_{3}}\right|_{\mathrm{p}, \infty, \mathrm{K}_{i}}^{2}\right\}^{1 / 2}
\end{array}\right\}
$$


Taking into account that the length of $\mathrm{k}_{i}$ is bounded by $h, 1 \leq i \leq M_{h}$, the results of interpolation theory in Sobolev spaces show that

$$
\left|v_{h j}\right|_{p, \infty, K_{i}} \leq C h^{-1 / 2}\left|v_{h j}\right|_{p, K_{i}}, j=1 \text { or } 3, p=0 \text { or } 1 \text {, }
$$

so that $(4.2 .23)$ yields

$$
\left|\tilde{\gamma}_{h_{1}}{ }^{1}\left(\vec{v}_{h}\right)-\gamma_{1}^{1}\left(\vec{v}_{h}\right)\right|_{0, K_{i}} \leq C h\left\{\left\|v_{h_{1}}\right\|_{1, K_{i}}^{2}+\left\|v_{h_{3}}\right\|_{2, K_{i}}^{2}\right\}^{1 / 2},
$$

which is exactly the estimate (4.2.2) .

Let us prove now the following theorem :

Theorem 4.2.2. : There exists a constant $c$, independent of $h$, such

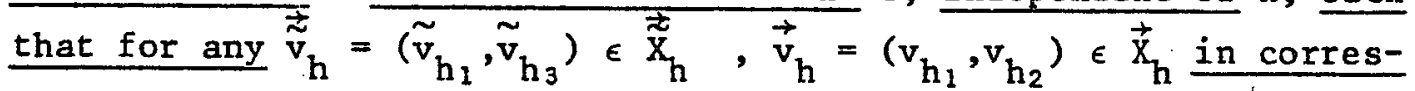
pondence through the bijection $F_{h}$ defined in Theorem 3.2.1', we have for any $i, 1 \leq i \leq M_{h}$ :

$$
\left|\tilde{\rho}_{h_{1}}{ }^{1}\left(\vec{v}_{h}\right)-\rho_{1}^{1}\left(\vec{v}_{h}\right)\right|_{0, K_{i}} \leq \operatorname{Ch}\left\{\left\|v_{h_{1}}\right\|_{1, k_{i}}^{2}+\left\|v_{h_{3}}\right\|_{2,}^{2}, k_{i}\right\}^{1 / 2}
$$

Proof (in six steps):

Step 1 : An expression of $\tilde{\rho}_{h}^{1}\left(\vec{v}_{h}\right)-\rho_{1}^{1}\left(\vec{v}_{h}\right)$ as function of $\vec{v}_{h}$

First, let us record that, from $(1.1 .17)$ and (3.3.2), we have :

$$
\rho_{1}^{1}\left(\vec{v}_{h^{\prime}}\right)=\frac{1}{\sqrt{a}}\left(\frac{1}{\sqrt{a}}\left(v_{h_{3}}^{\prime}-\frac{1}{R} v_{h_{1}}\right)^{\prime}, \tilde{\rho}_{h_{1}}{ }^{1}\left(\vec{v}_{h^{\prime}}\right)=\frac{1}{a_{h}} \tilde{v}_{h_{3}}^{\prime \prime} .\right.
$$


We still denote $\vec{\phi}(\xi)$ for $\vec{\phi}(\xi, 0)$. Then, if we set

$$
\begin{aligned}
& \psi\left(\vec{v}_{h}\right)=\frac{1}{\sqrt{a}}\left\{v_{h_{3}}^{\prime \prime}-\frac{1}{R} v_{h_{1}}^{\prime}+\frac{R^{\prime}}{R^{2}} v_{h_{1}}-\right. \\
& \left.\quad-\frac{\vec{\Phi}^{\prime} \cdot \vec{\phi}^{\prime \prime}}{a} v_{h_{3}}^{\prime}+\frac{1}{R} \frac{\vec{\Phi}^{\prime} \cdot \vec{\phi}^{\prime \prime}}{a} v_{h_{1}}\right\} \\
& \tilde{\psi}\left(\overrightarrow{\tilde{v}}_{h}\right)=\frac{1}{\sqrt{a_{h}}} \tilde{v}_{h_{3}}^{\prime \prime},
\end{aligned}
$$

we can write

$$
\left.\begin{array}{c}
\left.\tilde{\rho}_{h_{1}}^{1}\left(\vec{v}_{h}\right)-\rho_{1}^{1}\left(\vec{v}_{h}\right)=\frac{1}{\sqrt{a_{h}}}\left(\tilde{\psi}_{\left(v_{h}\right.}\right)-\psi\left(\vec{v}_{h}\right)\right)+ \\
+\left(\frac{1}{\sqrt{a_{h}}}-\frac{1}{\sqrt{a}}\right) \psi\left(\vec{v}_{h}\right) .
\end{array}\right\}
$$

$\frac{\text { Step 2 }}{\text { freedom of } \overrightarrow{\overrightarrow{\mathrm{x}}}_{\mathrm{h}} \text {. An expression of }} \tilde{\mathrm{v}}_{\mathrm{h}_{3}}^{\prime \prime}(\xi)$ as function of the degrees of From now on, let us consider the restriction $\overrightarrow{\widetilde{v}}_{h} / k_{i}$ to
any $\left.k_{i}=\right] \xi_{i-1}, \xi_{i}\left[\right.$. For simplicity, we still denote $\overrightarrow{\widetilde{v}}_{h}$ this
restriction. By definition of the space $\tilde{\tilde{x}}_{h_{2}}$, we have :

$$
\begin{aligned}
\tilde{v}_{h_{3}}(\xi)= & (1-\lambda)^{2}(1+2 \lambda) \tilde{v}_{h_{3}}\left(\xi_{i-1}\right)+\lambda^{2}(3-2 \lambda) \tilde{v}_{h_{3}}\left(\xi_{i}\right)+ \\
& +\lambda(1-\lambda)^{2}\left(\xi_{i}-\xi_{i-1}\right) \tilde{v}_{h_{3}}^{\prime}\left(\xi_{i-1}\right)+ \\
& +\lambda^{2}(\lambda-1)\left(\xi_{i}-\xi_{i-1}\right) \tilde{v}_{h_{3}}^{\prime}\left(\xi_{i}\right)
\end{aligned}
$$

with $\lambda=\frac{\xi-\xi_{i-1}}{\xi_{i}-\xi_{i-1}}$ 
Then, the first and second derivatives of $\dot{\mathrm{v}}_{\mathrm{h}_{3}}$ respectively admit the following expressions :

$$
\begin{aligned}
& \tilde{v}_{h_{3}}^{\prime}(\xi)=\frac{1}{\xi_{i}-\xi_{i-1}}\left(6 \lambda-6 \lambda^{2}\right)\left(\tilde{v}_{h_{3}}\left(\xi_{i}\right)-\tilde{v}_{h_{3}}\left(\xi_{i-1}\right)\right)+ \\
& +\left(3 \lambda^{2}-4 \lambda+1\right) \tilde{\nabla}_{h_{3}}^{\prime}\left(\xi_{i-1}\right)+ \\
& +\left(3 \lambda^{2}-2 \lambda\right) \tilde{\mathrm{v}}_{\mathrm{h}_{3}}^{\prime}\left(\xi_{i}\right) \\
& \tilde{v}_{h_{3}}^{\prime \prime}(\xi)=\frac{1}{\left(\xi_{i}-\xi_{i-1}\right)^{2}}(-12 \lambda+6)\left(\tilde{v}_{h_{3}}\left(\xi_{i}\right),-\tilde{v}_{h_{3}}\left(\xi_{i-1}\right)\right)+ \\
& +\frac{1}{\xi_{i}-\xi_{i-1}}\left\{(6 \lambda-4) \tilde{v}_{h_{3}}^{\prime}\left(\xi_{i-1}\right)+\right. \\
& \left.+(6 \lambda-2) \tilde{v}_{h_{3}}^{\prime}\left(\xi_{i}\right)\right\}
\end{aligned}
$$

and, evidently, we obtain similar expressions for $v_{h_{3}}^{\prime}(\xi)$ and $v_{h_{3}}^{\prime \prime}(\xi)$.

Step 3: An expression of $\tilde{\rho}_{h_{1}^{1}}\left(\overrightarrow{\tilde{v}}_{\mathrm{h}}\right)-\rho_{1}^{1}\left(\vec{v}_{\mathrm{h}}\right)$ as function of $\overrightarrow{\mathrm{v}}_{\mathrm{h}}$.

The relations $(3.2 .11)$ involve

$$
\tilde{v}_{h_{3}}\left(\xi_{i}\right)=\left\{v_{h_{1}}\left(\xi_{i}\right) \vec{a}^{1}\left(\xi_{i}\right)+v_{h_{3}}\left(\xi_{i}\right) \vec{a}^{3}\left(\xi_{i}\right)\right\} \cdot \vec{a}_{h_{3}} .
$$

From relations $(1.1 .10)(3.1 .8)(3.1 .9)$ and $(4.2 .20)$, the previous relation can be rewritten as 


$$
\left.\begin{array}{rl}
\tilde{v}_{h_{3}}\left(\xi_{i}\right)=\frac{1}{\sqrt{a\left(\xi_{i}\right)}} & \frac{\vec{a}_{h_{1}}}{\sqrt{a_{h}}} \cdot\left\{v_{h_{3}}\left(\xi_{i}\right) \vec{a}_{1}\left(\xi_{i}\right)-\right. \\
& \left.-v_{h_{1}}\left(\xi_{i}\right) \vec{a}_{3}\left(\xi_{i}\right)\right\}
\end{array}\right\}
$$

and thus

$$
\begin{aligned}
\tilde{v}_{h_{3}}\left(\xi_{i}\right)-\tilde{v}_{h_{3}}\left(\xi_{i-1}\right)= & \frac{\vec{a}_{h_{1}}}{\sqrt{a_{h}}} \cdot\left\{\left[v_{h_{3}}\left(\xi_{i}\right) \frac{\vec{a}_{i}\left(\xi_{i}\right)}{\sqrt{a\left(\xi_{i}\right)}}-\right.\right. \\
& \left.-v_{h_{3}}\left(\xi_{i-1}\right) \frac{\vec{a}_{1}\left(\xi_{i-1}\right)}{\sqrt{a\left(\xi_{i-1}\right)}}\right]- \\
& \left.-\left[v_{h_{1}}\left(\xi_{i}\right) \frac{\vec{a}_{3}\left(\xi_{i}\right)}{\sqrt{a\left(\xi_{i}\right)}}-v_{h_{1}}\left(\xi_{i-1}\right) \frac{\vec{a}_{3}\left(\xi_{i-1}\right)}{\sqrt{a\left(\xi_{i-1}\right)}}\right]\right\}
\end{aligned}
$$

From compatibility relation (3.2.9) and relation (3.2.14), we deduce

$$
\tilde{v}_{h_{3}}^{\prime}\left(\xi_{i}\right)=\frac{\sqrt{a_{h}}}{\sqrt{a\left(\xi_{i}\right)}}\left(v_{h_{3}}^{\prime}\left(\xi_{i}\right)-\frac{1}{R\left(\xi_{i}\right)} v_{h_{1}}\left(\xi_{i}\right)\right) \text {. }
$$

By substituting relations $(4.2 .28)$ to $(4.2 .30)$ into the relation $(4.2 .26)$ we find

$$
\begin{aligned}
& \tilde{\rho}_{h_{1}{ }^{1}}\left(\overrightarrow{\tilde{v}}_{h}\right)-\rho_{1}^{1}\left(\vec{v}_{h}\right)=\frac{1}{\sqrt{a_{h}}} \cdot\left(A\left(v_{h_{3}}\right)+B\left(v_{h_{1}}\right)\right)+ \\
& +\left(\frac{1}{\sqrt{a_{h}}}-\frac{1}{\sqrt{a}}\right) \psi\left(\vec{v}_{h}\right)
\end{aligned}
$$


$-35-$

where

$$
\begin{aligned}
A\left(v_{h_{3}}\right)= & \frac{1}{\left(\xi_{i}-\xi_{i-1}\right)^{2}}(-12 \lambda+6)\left\{\left[\frac{\vec{a}_{h_{1}}}{a_{h}} \cdot \frac{\vec{a}_{1}\left(\xi_{i}\right)}{\sqrt{a\left(\xi_{i}\right)}}-\frac{1}{\sqrt{a(\xi)}}\right] v_{h_{3}}\left(\xi_{i}\right)-\right. \\
& \left.-\left[\frac{a_{h_{1}}}{a_{h}} \cdot \frac{\overrightarrow{a_{1}}\left(\xi_{i-1}\right)}{\sqrt{a\left(\xi_{i-1}\right)}}-\frac{1}{\sqrt{a(\xi)}}\right] v_{h_{3}}\left(\xi_{i-1}\right)\right\}+ \\
& +\frac{1}{\left(\xi_{i}-\xi_{i-1}\right)}\left\{(6 \lambda-4)\left[\frac{1}{\sqrt{a\left(\xi_{i-1}\right)}}-\frac{1}{\sqrt{a(\xi)}}\right] v_{h_{3}}^{\prime}\left(\xi_{i-1}\right)+\right. \\
& \left.+(6 \lambda-2)\left[\frac{1}{\sqrt{a\left(\xi_{i}\right)}}-\frac{1}{\sqrt{a(\xi)}}\right] v_{h_{3}}^{\prime}\left(\xi_{i}\right)\right\}+\frac{1}{\sqrt{a(\xi)}} \frac{\vec{\Phi}^{\prime}(\xi) \cdot \dot{\phi}^{\prime \prime}(\xi)}{a(\xi)} v_{h_{3}}^{\prime}
\end{aligned}
$$

and

$$
\begin{aligned}
& B\left(v_{h_{1}}\right)= \frac{1}{\sqrt{a_{h}}}\left\{\frac{-1}{\left(\xi_{i}-\xi_{i-1}\right)^{2}}(-12 \lambda+6) \frac{\vec{a}_{h_{1}}}{\sqrt{a_{h}}} \cdot\right. \\
& \cdot\left[v_{h_{1}}\left(\xi_{i}\right) \frac{\vec{a}_{3}\left(\xi_{i}\right)}{\sqrt{a\left(\xi_{i}\right)}}-v_{h_{i}}\left(\xi_{i-1}\right) \frac{\vec{a}_{3}\left(\xi_{i-1}\right)}{\sqrt{a\left(\xi_{i-1}\right)}}\right] \\
&-\frac{1}{\xi_{i}-\xi_{i-1}}\left[(6 \lambda-4) \frac{\sqrt{a_{h}}}{\sqrt{a\left(\xi_{i-1}\right)}} \frac{1}{R\left(\xi_{i-1}\right)} v_{h_{1}}\left(\xi_{i-1}\right)+\right. \\
&+(6 \lambda-2) \frac{\sqrt{a_{h}}}{\left.\left.\sqrt{a\left(\xi_{i}\right)} \frac{1}{R\left(\xi_{i}\right)} v_{h_{1}}\left(\xi_{i}\right)\right]\right\}-} \\
&-\frac{1}{\sqrt{a(\xi)}}\left\{\frac{R^{\prime}(\xi)}{(R(\xi))^{2}} v_{h_{1}}(\xi)+\frac{1}{R(\xi)} \frac{\vec{\Phi}^{\prime}(\xi) \cdot \vec{\phi}^{\prime \prime}(\xi)}{a(\xi)} v_{h_{1}}(\xi)-\frac{1}{R(\xi)} v_{h_{1}}^{\prime}(\xi)\right\}
\end{aligned}
$$


Step 4: Finite expansion of $\mathrm{A}\left(\mathrm{v}_{\mathrm{h}_{3}}\right)$.

We have the following finite expansions

$$
\begin{aligned}
\vec{a}_{1}\left(\xi_{i}\right)= & \vec{\phi}^{\prime}(\xi)+\left(\xi_{i}-\xi\right) \vec{\phi}^{\prime \prime}(\xi)+\frac{1}{2}\left(\xi_{i}-\xi\right)^{2} \vec{\phi}^{\prime \prime \prime}(\xi)+o\left(h^{3}\right) \\
\frac{1}{\sqrt{a\left(\xi_{i}\right)}}= & \frac{1}{\sqrt{a(\xi)}}\left\{1-\left(\xi_{i}-\xi\right) \frac{\vec{\phi}^{\prime}(\xi) \cdot \vec{\phi}^{\prime \prime}(\xi)}{a(\xi)}-\right. \\
& -\frac{1}{2} \frac{\left(\xi_{i}-\xi\right)^{2}}{a(\xi)}\left(\vec{\phi}^{\prime \prime}(\xi) \cdot \vec{\phi}^{\prime \prime}(\xi)+\vec{\phi}^{\prime}(\xi) \cdot \vec{\phi}^{\prime \prime \prime}(\xi)\right)+ \\
& \left.+\frac{3}{2} \frac{\left(\xi_{i}-\xi\right)^{2}}{[a(\xi)]^{2}}\left(\vec{\phi}^{\prime}(\xi) \cdot \vec{\phi}^{\prime \prime}(\xi)\right)^{2}\right\}+o\left(h^{3}\right)
\end{aligned}
$$

and thus

$$
\begin{aligned}
\frac{\vec{a}_{1}\left(\xi_{i}\right)}{\sqrt{a\left(\xi_{i}\right)}}= & \frac{1}{\sqrt{a(\xi)}}\left\{\vec{\phi}^{\prime}(\xi)+\left(\xi_{i}-\xi\right)\left[\vec{\phi}^{\prime \prime}(\xi)-\right.\right. \\
& \left.-\frac{\vec{\Phi}^{\prime}(\xi) \cdot \vec{\phi}^{\prime \prime}(\xi)}{a(\xi)} \vec{\phi}^{\prime}(\xi)\right]+\frac{1}{2}\left(\xi_{i}-\xi\right)^{2}\left[\vec{\phi}^{\prime \prime \prime}(\xi)-\right. \\
& -\frac{\vec{\phi}^{\prime \prime}(\xi) \cdot \vec{\phi}^{\prime \prime}(\xi)}{a(\xi)} \vec{\phi}^{\prime}(\xi)-\frac{\vec{\phi}^{\prime}(\xi) \cdot \vec{\phi}^{\prime \prime \prime}(\xi)}{a(\xi)} \vec{\phi}^{\prime}(\xi) \\
& +3 \frac{\left(\vec{\phi}^{\prime}(\xi) \cdot \vec{\phi}^{\prime \prime}(\xi)\right)^{2}}{[a(\xi)]^{2}} \vec{\phi}^{\prime}(\xi)- \\
& \left.\left.-2 \frac{\vec{\phi}^{\prime}(\xi) \cdot \vec{\phi}^{\prime \prime}(\xi)}{a(\xi)} \vec{\phi}^{\prime \prime}(\xi)\right]\right\}+o\left(h^{3}\right)
\end{aligned}
$$


From expression (3.1.8), it follows

$$
\left.\begin{array}{rl}
\vec{a}_{h_{1}}=\vec{\phi}^{\prime}(\xi) & +\frac{1}{2}\left(\xi_{i}+\xi_{i-1}-2 \xi\right) \vec{\phi}^{\prime \prime}(\xi)+\frac{1}{6}\left[\left(\xi_{i}-\xi\right)^{2}+\right. \\
& \left.+\left(\xi_{i}-\xi\right)\left(\xi_{i-1}-\xi\right)+\left(\xi_{i-1}-\xi\right)^{2}\right] \vec{\phi}^{\prime \prime \prime}(\xi)+o\left(h^{3}\right)
\end{array}\right\}(4.2 .35)
$$

Since

$$
\begin{aligned}
& a_{h}=a(\xi)+\left(\xi_{i}+\xi_{i-1}-2 \xi\right) \vec{\phi}^{\prime}(\xi) \cdot \vec{\phi}^{\prime \prime}(\xi)+ \\
& +\frac{1}{4}\left(\xi_{i}+\xi_{i-1}-2 \xi\right)^{2} \vec{\phi}^{\prime \prime}(\xi) \cdot \vec{\phi}^{\prime \prime}(\xi)+ \\
& +\frac{1}{3}\left[\left(\xi_{i}-\xi\right)^{2}+\left(\xi_{i}-\xi\right)\left(\xi_{i-1}-\xi\right)+\left(\xi_{i-1}-\xi\right)^{2}\right] \vec{\phi}^{\prime}(\xi) \cdot \vec{\phi}^{\prime \prime \prime}(\xi) \\
& +o\left(h^{3}\right)
\end{aligned}
$$

we find

$$
\begin{aligned}
\frac{1}{a_{h}}=\frac{1}{a(\xi)}\left\{1-\left(\xi_{i}+\xi_{i-1}-2 \xi\right) \frac{\vec{\Phi}^{\prime}(\xi) \cdot \vec{\Phi}^{\prime \prime}(\xi)}{a(\xi)}-\right. \\
-\frac{1}{4}\left(\xi_{i}+\xi_{i-1}-2 \xi\right)^{2} \frac{\vec{\Phi}^{\prime \prime}(\xi) \cdot \vec{\phi}^{\prime \prime}(\xi)}{a(\xi)}+ \\
+\left(\xi_{i}+\xi_{i-1}-2 \xi\right)^{2} \frac{\left(\vec{\phi}^{\prime}(\xi) \cdot \vec{\phi}^{\prime \prime}(\xi)\right)^{2}}{[a(\xi)]^{2}}- \\
-\frac{1}{3}\left[\left(\xi_{i}-\xi\right)^{2}+\left(\xi_{i}-\xi\right)(\xi)-\xi\right)+ \\
\left.\left.+\left(\xi_{i-1}-\xi\right)^{2}\right] \frac{\vec{\phi}^{\prime}(\xi) \cdot \dot{\phi}^{\prime \prime \prime}(\xi)}{a(\xi)}\right\}+0\left(h^{3}\right)
\end{aligned}
$$


Consequently, we obtain, on the one hand,

$$
\begin{gathered}
\frac{\vec{a}_{h_{1}}}{a_{h}} \cdot \frac{\vec{a}_{1}\left(\xi_{i}\right)}{\sqrt{a\left(\xi_{i}\right)}}-\frac{1}{\sqrt{a(\xi)}}=\frac{1}{\sqrt{a(\xi)}}\left\{-\frac{1}{2}\left(\xi_{i}+\xi_{i-1}-2 \xi\right) \frac{\vec{\phi}^{\prime}(\xi) \cdot \dot{\phi}^{\prime \prime}(\xi)}{a(\xi)}+\right. \\
+\Phi(\xi)\}+o\left(h^{3}\right)
\end{gathered}
$$

where

$$
\begin{aligned}
& \Phi(\xi)=\frac{1}{2}\left(\xi_{i}+\xi_{i-1}-2 \xi\right)^{2}\left[\frac{\left(\vec{\phi}^{\prime}(\xi): \vec{\phi}^{\prime \prime}(\xi)\right)^{2}}{[a(\xi)]^{2}}-\frac{1}{2} \frac{\Phi^{\prime \prime}(\xi) \cdot \vec{\phi}^{\prime \prime}(\xi)}{a(\xi)}\right. \\
& -\frac{1}{6}\left[\left(\xi_{i}-\xi\right)^{2}+\left(\xi_{i}-\xi\right)\left(\xi_{i-1}-\xi\right)+\right. \\
& \left.+\left(\xi_{i-1}-\xi\right)^{2}\right] \frac{\vec{\Phi}^{\prime}(\xi) \cdot \vec{\phi}^{\prime \prime \prime}(\xi)}{a(\xi)}- \\
& -\frac{1}{2}\left(\xi_{i}-\xi\right)\left(\xi_{i-1}-\xi\right)\left[\frac{\left(\vec{\phi}^{\prime}(\xi) \cdot \dot{\phi}^{\prime \prime}(\xi)\right)^{2}}{[a(\xi)]^{2}}-\right. \\
& \left.-\frac{\vec{\Phi}^{\prime \prime}(\xi) \cdot \dot{\phi}^{\prime \prime}(\xi)}{a(\xi)}\right] \text {, }
\end{aligned}
$$

and, on the other hand,

$$
\frac{1}{\sqrt{a\left(\xi_{i}\right)}}-\frac{1}{\sqrt{a(\xi)}}=\frac{1}{\sqrt{a(\xi)}}\left\{-\left(\xi_{i}-\xi\right) \frac{\vec{\phi}^{\prime}(\xi) \cdot \vec{\phi}^{\prime \prime}(\xi)}{a(\xi)}+o\left(h^{2}\right)\right\}
$$

Let us observe that the first development is symetric with respect to $\xi_{i}$ and $\xi_{i-1}$. Then, by taking into account the expression $(4.2 .27)$, the relation $(4.2 .32)$ yields 


$$
\begin{aligned}
& A\left(v_{h_{3}}\right)=-\frac{\vec{\phi}^{\prime}(\xi) \cdot \vec{\phi}^{\prime}(\xi)}{a(\xi) \sqrt{a(\xi)}}\left\{-3 v_{h_{3}}^{\prime}(\xi)+\right. \\
& \left.+\frac{3}{\left(\xi_{i}-\xi_{i-1}\right.}\right)\left(v_{h_{3}}\left(\xi_{i}\right)-v_{h_{3}}\left(\xi_{i-1}\right)\right)+ \\
& \left.+2(2 \lambda-1)\left(v_{h_{3}}^{\prime}\left(\xi_{i}\right)-v_{h_{3}}^{\prime}\left(\xi_{i-1}\right)\right)\right\}+ \\
& +\frac{(-12 \lambda+6)}{\left(\xi_{i}-\xi_{i-1}\right)^{2}}\left(v_{h_{3}}\left(\xi_{i}\right)-v_{h_{3}}\left(\xi_{i-1}\right)\right) \frac{\Phi(\xi)}{\sqrt{a(\xi)}}+ \\
& +\frac{(-12 \lambda+6)}{\left(\xi_{i}-\xi_{i-1}\right)^{2}}\left[0\left(h^{3}\right) v_{h_{3}}\left(\xi_{i}\right)+o\left(h^{3}\right) v_{h_{3}}\left(\xi_{i-1}\right)\right]+ \\
& +\frac{1}{\xi_{i}-\xi_{i-1}}\left\{0\left(h^{2}\right)(6 \lambda-4) v_{h_{3}}^{\prime}\left(\xi_{i-1}\right)+\right. \\
& \left.+o\left(h^{2}\right)(6 \lambda-2) v_{h_{3}}^{\prime}\left(\xi_{i}\right)\right\} \quad .
\end{aligned}
$$

But Taylor's formula implies

$$
\begin{aligned}
& v_{h_{3}}\left(\xi_{i}\right)-v_{h_{3}}\left(\xi_{i-1}\right)=\left(\xi_{i}-\xi_{i-1}^{\prime}\right) v_{h_{3}}^{\prime}(\theta), \xi_{i-1} \leq \theta \leq \xi_{i}, \\
& v_{h_{3}}^{\prime}\left(\xi_{i}\right)-v_{h_{3}}^{\prime}\left(\xi_{i-1}\right)=\left(\xi_{i}-\xi_{i-1}\right) v_{h_{3}}^{\prime \prime}(n), \xi_{i-1} \leq n \leq \xi_{i}, \\
& \quad . \\
& v_{h_{3}}^{\prime}\left(\xi_{)}-\frac{1}{\xi_{i}-\xi_{i-1}}\left(v_{h_{3}}\left(\xi_{i}\right)-v_{h_{3}}\left(\xi_{i-1}\right)\right)=0(h) v_{h_{3}}^{\prime \prime}(\omega), \xi_{i-1} \leq \omega \leq \xi_{i}\right.
\end{aligned}
$$

Since $\Phi(\xi)=O\left(h^{2}\right)$, there exist $\theta^{j} \in\left[\xi_{i-1}, \xi_{i}\right], j=0,1,2$

such that

$$
A\left(v_{h_{3}}\right)=O(h)\left(\sum_{j=0}^{2} v_{h_{3}}^{(j)}\left(\theta^{j}\right)\right)
$$




\section{Step 5 : Finite expansion of $B\left(v_{h_{1}}\right)$.}

We have

$$
\left.\begin{array}{l}
\vec{a}_{3}\left(\xi_{i}\right)=\vec{a}_{3}(\xi)+\left(\xi_{i}-\xi\right) \vec{a}_{3}^{\prime}(\xi)+\frac{1}{2}\left(\xi_{i}-\xi\right)^{2} \vec{a}_{3}^{\prime \prime}(\xi)+O\left(h^{3}\right), \\
v_{h_{2}}\left(\xi_{i}\right)=v_{h_{1}}(\xi)+\left(\xi_{i}-\xi\right) v_{h_{1}}^{\prime} .
\end{array}\right\}
$$

Since $\vec{a}_{3}(\xi) \cdot \vec{\phi}^{\prime}(\xi)=0$, we obtain with $(4.2 .16)$

$$
\begin{aligned}
& \vec{a}_{3}^{\prime}(\xi) \cdot \vec{\phi}^{\prime}(\xi)=-\vec{a}_{3}(\xi) \cdot \vec{\phi}^{\prime \prime}(\xi)=\frac{a(\xi)}{R(\xi)}, \\
& \vec{a}_{3}^{\prime \prime}(\xi) \cdot \vec{\phi}^{\prime}(\xi)+\vec{a}_{3}^{\prime}(\xi) \cdot \vec{\phi}^{\prime \prime}(\xi)=\left(\frac{a(\xi)}{R(\xi)}\right)^{\prime} \cdot
\end{aligned}
$$

Combining these last relations with finite expansions $(4.2 .34)(4.2 .35)(4.2 .36)$ and $(4.2 .38)$, we get for any $\xi \in\left[\xi_{i-1}, \xi_{i}\right]$

$$
\begin{aligned}
\frac{\vec{a}_{h_{1}}}{a_{h}}\left[v_{h_{1}}\left(\xi_{i}\right)\right. & \left.\frac{\vec{a}_{3}\left(\xi_{i}\right)}{\sqrt{a\left(\xi_{i}\right)}}-v_{h_{1}}\left(\xi_{i-1}\right) \frac{\vec{a}_{3}\left(\xi_{i-1}\right)}{\sqrt{a\left(\xi_{i-1}\right)}}\right]= \\
& =\frac{\left(\xi_{i}-\xi_{i-1}\right)}{\sqrt{a(\xi)}}\left\{\frac{1}{R(\xi)} v_{h_{1}}(\xi)+\right. \\
& +\frac{1}{2}\left(\xi_{i}+\xi_{i-1}-2 \xi\right)\left[\frac{1}{R(\xi)} v_{h_{1}}^{\prime}(\xi)-\right. \\
& \left.\left.-\frac{R^{\prime}(\xi)}{(R(\xi))^{2}} v_{h_{1}}(\xi)-\frac{1}{R(\xi)} \frac{\vec{\phi}^{\prime}(\xi) \cdot \dot{\phi}^{\prime \prime}(\xi)}{a(\xi)} v_{h_{1}}(\xi)\right]\right\}+ \\
& +\left(v_{h_{1}}(\xi)+v_{h_{1}^{\prime}}^{\prime}\right) o\left(h^{3}\right) .
\end{aligned}
$$


Next, we have

$$
R\left(\xi_{i}\right)=R(\xi)+\left(\xi_{i}-\xi\right) R^{\prime}(\xi)+o\left(h^{2}\right)
$$

so that

$$
\frac{1}{R\left(\xi_{i}\right)}=\frac{1}{R(\xi)}\left\{1-\left(\xi_{i}-\xi\right) \frac{R^{\prime}(\xi)}{R(\xi)}+0\left(h^{2}\right)\right\} .
$$

By using ( 4.2 .34$)$, we obtain

$$
\begin{aligned}
& \frac{1}{R\left(\xi_{i}\right)} \frac{1}{\sqrt{a\left(\xi_{i}\right)}} v_{h_{1}}\left(\xi_{i}\right)=\frac{1}{\sqrt{a(\xi)}} \frac{1}{R(\xi)}\left\{v_{h_{1}}(\xi)+\right. \\
& +\left(\xi_{i}-\xi\right)\left[v_{h_{1}}^{\prime}(\xi)-\frac{R^{\prime}(\xi)}{R(\xi)} v_{h_{1}}(\xi)-\right. \\
& \left.\left.-\frac{\dot{\Phi}^{\prime}(\xi) \cdot \dot{\phi}^{\prime \prime}(\xi)}{a(\xi)} v_{h_{1}}(\xi)\right]\right\}+ \\
& +\left(v_{h_{1}}(\xi)+v_{h_{1}^{\prime}}^{\prime}\right) O\left(h^{2}\right) .
\end{aligned}
$$

By combining this last finite expansion with (4.2.39), the expression (4.2.33) involves :

$$
B\left(v_{h_{1}}\right)=O(h)\left(\sum_{j=0}^{1} v_{h_{1}}^{(j)}\left(\theta^{j}\right)\right), \xi_{i-1} \leq \theta^{j} \leq \xi_{i} \cdot(4.2 .40)
$$


Step 6: Obtention of estimate $(4: 2.24)$.

From $(4.2 .36)$, it follows

$\frac{1}{\sqrt{a_{h}}}=\frac{1}{\sqrt{a(\xi)}}\{1+0(h)\}$.

By using the last expression combined with finite expansions $(4.2 .37)$ and $(4.2 .40)$, we prove from $(4.2 .25)$ and (4.2.31), the existence of values $\theta^{j} \in\left[\xi_{i-1}, \xi_{i}\right], j=0,1$ and $n^{j} \in\left[\xi_{i-1}, \xi_{i}\right], j=0,1,2$ such that

$$
\left.\begin{array}{c}
\tilde{\rho}_{h_{1}}^{i}\left(\underset{v_{h}}{\vec{v}_{h}}-\rho_{1}^{1}\left(\vec{v}_{h}\right)=o(h)\left[\sum _ { j = 0 } ^ { 1 } \left\{v_{h_{1}}^{(j)}\left(\theta^{j}\right)+\right.\right.\right. \\
\left.\left.+v_{h_{3}}^{(j)}\left(n^{j}\right)\right\}+v_{h_{3}}^{\prime \prime}\left(n^{2}\right)\right]
\end{array}\right\}
$$

Then, we derive for any element $k_{i}$ of $\mathscr{C}_{h}$ the existence of a constant $\dot{C}$ independent of $h$ such that

$$
\begin{gathered}
\left|\tilde{\rho}_{h_{1}{ }^{1}}\left(\overrightarrow{\tilde{v}}_{h}\right)-\rho_{1}^{1}\left(\vec{v}_{h}\right)\right|_{0, K_{i}} \leq c^{3} /\left.2\left|\sum_{p=0}^{1}\right| v_{h_{1}}\right|_{p, \infty, K_{i}} ^{2}+ \\
\left.+\sum_{p=0}^{2}\left|v_{h_{3}}\right|_{p, \infty, K_{i}}^{2}\right\}^{1 / 2} .
\end{gathered}
$$

The estimate $(4.2 .24)$ is obtained by using similar arguments to those developped at the end of the proof of Theorem 4.2.1. 
Combining the previous theorems, we obtain

Theorem 4:2.3. : There exists a constant $C$ independent of $h$ such that for any $\vec{v}_{h}, \vec{w}_{h} \in \overrightarrow{\mathrm{x}}_{h}$ and any $\overrightarrow{\mathrm{v}}_{\mathrm{h}}$, $\overrightarrow{\mathrm{w}}_{\mathrm{h}} \in \overrightarrow{\mathrm{X}}_{\mathrm{h}}$ in correspondence through the bijection $F_{h}$ proved in Theorem 3.2 .1 , we have

$$
\left|a\left(\vec{v}_{h}, \vec{w}_{h}\right)-\tilde{a}_{h}\left(\overrightarrow{\tilde{v}}_{h}, \overrightarrow{\tilde{w}}_{h}\right)\right| \leq C h\left\|\vec{v}_{h}\right\|\left\|\vec{w}_{h}\right\|
$$

Proof.: The relations $(1.2 .3)$ and $(3.3 .1)$ involve :

$$
\left|a\left(\vec{v}_{h}, \vec{w}_{h}\right)-\tilde{a}_{h}\left(\overrightarrow{\tilde{v}}_{h}, \overrightarrow{\tilde{w}}_{h}\right)\right| \leq \sum_{i=1}^{M_{h}}\left(\left|A_{i}\left(\vec{v}_{h}, \vec{w}_{h}\right)\right|+\left|B_{i}\left(\vec{v}_{h}, \vec{w}_{h}\right)\right|\right),
$$

with

$$
\begin{aligned}
& A_{i}\left(\vec{v}_{h}, \vec{w}_{h}\right)=\int_{K_{i}} \frac{E e}{1-v^{2}}\left\{\gamma_{l}^{1}\left(\vec{v}_{h}\right) \gamma_{l}^{1}\left(\vec{w}_{h}\right) \sqrt{a}-\right. \\
& \left.\left.-\tilde{\gamma}_{h_{1}}{ }^{1}\left(\overrightarrow{\tilde{v}}_{h}\right) \tilde{\gamma}_{h_{1}}{ }^{1} \overrightarrow{\tilde{w}}_{h}\right) \sqrt{a_{h}}\right\} d \xi \\
& B_{i}\left(\vec{v}_{h}, \vec{w}_{h}\right)=\int_{k_{i}} \frac{E e^{3}}{12\left(1-v^{2}\right)}\left\{\rho_{1}^{1}\left(\vec{v}_{h}\right) \rho_{i}^{1}\left(\vec{w}_{h}\right) \sqrt{a}-\right. \\
& \left.-\tilde{\rho}_{h I}^{1}\left(\overrightarrow{\tilde{v}}_{h}\right) \tilde{\rho}_{h_{1}}{ }^{1}\left(\overrightarrow{\tilde{w}}_{h}\right) \sqrt{a_{h}}\right\} \mathrm{d} \xi .
\end{aligned}
$$

From $(4.2 .21)$, we have for any $\xi \in K_{i}, 1 \leq i \leq M_{h}$,

$$
\sqrt{a}_{h}=\sqrt{a(\xi)}+O(h) \text {. }
$$


Moreover, it is easy to prove that for any $\vec{v}_{h} \in \vec{x}_{h}$ and any $\overrightarrow{\widetilde{v}}_{h} \in \overrightarrow{\tilde{x}}_{h}$ in correspondence through the bijection $F_{h}$, there exist constants $C_{1}>0$ and $C_{2}:>0$, independent of $h$, such that

$$
c_{1}\left\|\vec{v}_{h}\right\| \leq\left\|\vec{v}_{h}\right\|_{h} \leq c_{2}\left\|\vec{v}_{h}\right\|
$$

where $\|\cdot\|_{h}$ is defined by (3.2.15).

Combining $(4.2 .44)(4.2 .45)$ with estimates (4.2.2) and (4.2.24), we derive the existence of constants $C_{1}$ and $C_{2}$ independent of $h$ such that

$$
\begin{aligned}
& \left|A_{i}\left(\vec{v}_{h}, \vec{w}_{h}\right)\right| \leq c_{i} h\left\|\vec{v}_{h}\right\|_{K_{i}}\left\|\vec{w}_{h}\right\|_{K_{i}}, 1 \leq i \leq M_{h}, \\
& \left|B_{i}\left(\vec{v}_{h}, \vec{w}_{h}\right)\right| \leq C_{2} h\left\|\vec{v}_{h}\right\|_{K_{i}}\left\|\vec{w}_{h}\right\|_{K_{i}}, 1 \leq i \leq M_{h} .
\end{aligned}
$$

Estimation $(4.2 .43)$ follows by summation over $i, \quad l \leq i \leq M_{h}$.

Now, we consider the estimate of the difference $\left|\tilde{a}_{h}\left(\overrightarrow{\tilde{v}}_{h}, \overrightarrow{\tilde{w}}_{h}\right)-a_{h}^{*}\left(\overrightarrow{\tilde{v}}_{h}, \vec{\sim}_{h}\right)\right|$ which appears in relation $(4.2 .1)$.

By using $(3.3 .1)$ and $(3.3 .11)$, we get for any $\overrightarrow{\tilde{v}}_{h}, \overrightarrow{\tilde{w}}_{h} \in \overrightarrow{\tilde{x}}_{h}$ :

$$
\left.\begin{array}{rl}
\tilde{a}_{h}\left(\overrightarrow{\tilde{v}}_{h}, \overrightarrow{\tilde{w}}_{h}\right)-a_{h}^{*}\left(\overrightarrow{\tilde{v}}_{h}, \vec{\sim}_{h}\right) & \\
& =\sum_{i=1}^{M_{h}}\left\{E_{k_{i}}^{1} \underset{\left.\vec{w}_{h}, \vec{w}_{h}\right)+E_{K_{i}}^{2}}{\left(\vec{v}_{h}, \overrightarrow{\tilde{w}}_{h}\right)}\right\}
\end{array}\right\}
$$


where

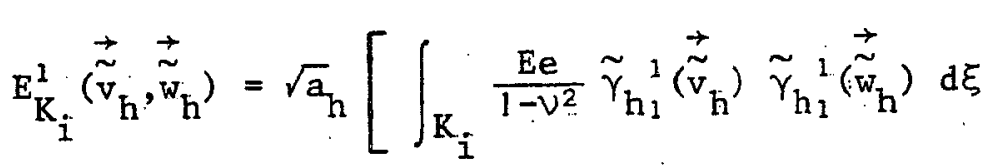

$$
\begin{aligned}
& \left.\left.\left.-\sum_{\ell_{1}=1}^{L} \omega_{\ell_{1}, K_{i}}^{L}\left\{\frac{E e}{1-v^{2}} \tilde{\gamma}_{h_{1}}{ }^{1} \overrightarrow{\tilde{v}}_{h}\right), \tilde{\gamma}_{h_{1}}{ }^{1}\left(\overrightarrow{\tilde{w}}_{h}\right)\right\}\left\{b_{\ell_{1}, K_{i}}\right\}\right]\right\}
\end{aligned}
$$

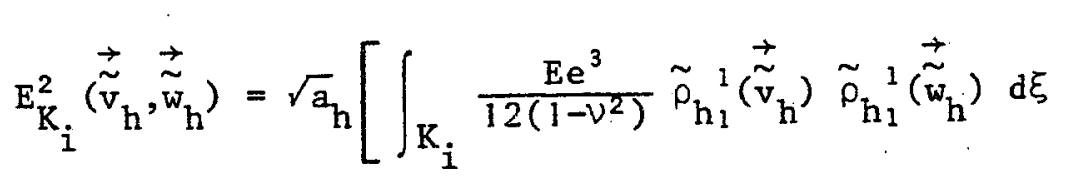

$$
\begin{aligned}
& \left.-\sum_{\ell_{2}=1}^{\mathrm{L}_{2}} \omega_{\ell_{2}, \mathrm{k}_{i}}\left\{\frac{E e^{3}}{12\left(1-v^{2}\right)} \tilde{\rho}_{\mathrm{h}_{1}}{ }^{1}\left(\overrightarrow{\tilde{v}}_{\mathrm{h}}\right) \tilde{\rho}_{\mathrm{h}_{1}}{ }^{1}\left(\vec{\sim}_{\mathrm{h}}\right)\right\}\left\{\mathrm{b}_{\ell_{2}, \mathrm{~K}_{i}}\right\}\right\}
\end{aligned}
$$

Then, we prove the following theorem:

Theorem 4.2.4.: Assume that the numerical integration schemes which appear in $(3.3 .11)(4.2 .47)(4.2 .48)$ are derived from schemes defined over the reference element $K$ and such that

$$
\begin{aligned}
& \forall \hat{\phi} \in \mathrm{P}_{\theta}(\hat{\mathrm{K}}) \quad, \hat{\mathrm{E}}^{1}(\hat{\phi})=0, \\
& \forall \hat{\phi} \in \dot{\mathrm{P}}_{2}(\hat{\mathrm{K}}) \quad, \hat{\mathrm{E}}^{2}(\hat{\phi})=0 .
\end{aligned}
$$

Here, $\hat{\mathrm{E}}^{\alpha}$ is associated to $\mathrm{E}_{\mathrm{K}_{\mathrm{i}}}^{\alpha}$ through relations (3.3.8) to $(3.3 .10)$, i.e.,

$$
\hat{\mathbf{E}}^{\alpha}(\hat{\phi})=\int_{\hat{\mathrm{K}}} \hat{\phi}(\hat{\mathrm{x}}) \mathrm{d} \hat{\mathrm{x}}-\sum_{\ell_{\alpha}=1}^{\mathrm{L}_{\alpha}} \hat{\omega}_{\ell_{\alpha}} \hat{\phi}\left(\hat{\mathrm{b}}_{\ell_{\alpha}}\right), \alpha=1 \text { or } 2 \text {. }
$$


Then, for any regular partition $\mathscr{C}_{\mathrm{h}}$ of $\overline{\mathrm{I}}$ (see $(2.1 .1)$ ) there exists a constant $C$, independent of $h$, such that

$$
\left|\tilde{a}_{h}\left(\overrightarrow{\tilde{v}}_{h}, \overrightarrow{\tilde{w}}_{h}\right)-a_{h}^{*}\left(\overrightarrow{\tilde{v}}_{h}, \overrightarrow{\tilde{w}}_{h}\right)\right| \leq \mathrm{Ch}\left\|\overrightarrow{\tilde{v}}_{h}\right\|_{h}\left\|\overrightarrow{\tilde{w}}_{h}\right\|_{h}
$$

$\frac{\text { Proof }}{\underset{\sim}{\vec{\sim}}}$ : For simplicity, we have denoted $\mathrm{E}_{\mathrm{K}_{i}}^{1}$ as a function of $\widetilde{\mathrm{v}}_{\mathrm{h}}, \tilde{\mathrm{w}}_{\mathrm{h}}$. In fact, $\mathrm{E}_{\mathrm{K}_{i}}^{1}$ is just a function of $\tilde{\mathrm{v}}_{\mathrm{h}_{1}}$ and $\tilde{\mathrm{w}}_{\mathrm{h}_{1}}$. The corresponding function to integrate in $(4.2 .47)$ is the product of variable coefficient $\frac{E e}{1-v^{2}}$ by functions $\left.\tilde{\gamma}_{h_{1}}{ }^{1} \overrightarrow{\tilde{v}}_{h}\right)=\frac{1}{a_{h}} \tilde{v}_{h_{1}}^{\prime}(\xi)$. Then, by assuming that the variable coefficient $\frac{E e}{1-v^{2}}$ is sufficiently smooth, we obtain as a consequence of $[1$, part III, Theorem 4.4.1] or [2, Theorem 4.3]

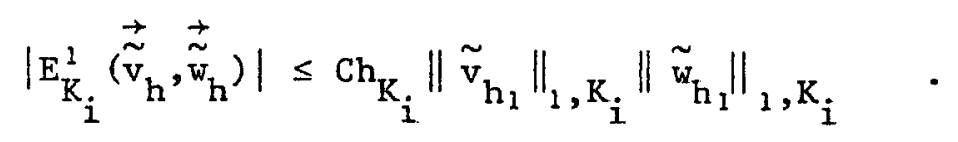

Similarly, we obtain

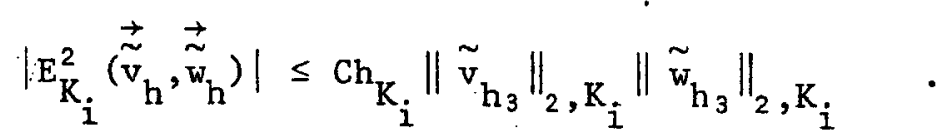

By combining above estimates with $(4.2 .46)$, we immediately obtain the estimate $(4 \cdot 2.52)$.

Combining the results of Theorems 4.2 .3 and 4.2 .4 , we finally find : 
Theorem 4.2.5. : Assume that the numerical integration schemes which appear in $(3.3 .11)(4.2 .47)(4.2 .48)$ are derived from schemes defined over the reference element $\hat{K}$ and such that

$$
\begin{aligned}
& \forall \hat{\phi} \in P_{0}(\hat{K}), \hat{E}^{1}(\hat{\phi})=0, \\
& \forall \hat{\phi} \in P_{2}(\hat{K}), \hat{E}^{2}(\hat{\phi})=0,
\end{aligned}
$$

with $\hat{\mathrm{E}}^{1}$ and $\hat{\mathrm{E}}^{2}$ defined by $(4.2 .51)$. Then, for any regular partition $\mathscr{E}_{h}$ of $\overline{\mathrm{I}}$ - see $(2: 1.1)$ - there exists a constant $\mathrm{C}$, independent of $h$, such that for any $\vec{v}_{h}, \vec{w}_{h} \in \overrightarrow{\mathrm{x}}_{h}$, we have

$$
\left|a\left(\vec{v}_{h}, \vec{w}_{h}\right)-b_{h}\left(\vec{v}_{h}, \vec{w}_{h}\right)\right| \leq C h\left\|\vec{v}_{h}\right\|\left\|\vec{w}_{h}\right\|
$$

where the bilinear forms $a(.,$.$) and b_{h}(.,$.$) are defined$ by $(1.2 .3)$ and $(3.3 .13)$.

\section{Proof :}

i) From Theorem 4.2.3, we have

$$
\left|a\left(\vec{v}_{h}, \vec{w}_{h}\right)-\tilde{a}_{h}\left(\overrightarrow{\tilde{v}}_{h}, \overrightarrow{\tilde{w}}_{h}\right)\right| \leq C h\left\|\vec{v}_{h}\right\|\left\|\vec{w}_{h}\right\| .
$$

ii) From Theorem 4.2 .4 and inequalities $(4.2 .45)$ we

have

$$
\left|\tilde{a}_{h}\left(\overrightarrow{\tilde{v}}_{h}, \overrightarrow{\tilde{w}}_{h}\right)-a_{h}^{*}\left(\overrightarrow{\tilde{v}}_{h}, \overrightarrow{\tilde{w}}_{h}\right)\right| \leq \mathrm{ch}\left\|\vec{v}_{h}\right\|\left\|\vec{w}_{h}\right\| \text {. }
$$


iii) Then, estimate $(4.2 .55)$ arises from above estimates and decomposition $(4.2 .1)$.

4.3 - Estimate of the consistency error $\left|f\left(\vec{w}_{h}\right)-f_{h}\left(\vec{w}_{h}\right)\right|$

Now, we prove the following theorem :

Theorem 4.3.1.: For any regular partition ${ }^{\circ}$ of $\bar{I}$ and for any components $p^{1}, p^{3} \in W^{1, q}(I), q \in \mathbb{R}, q \geq 2$, there exists a constant $C$, independent of $\mathrm{h}$, such that

$$
\left|f\left(\vec{w}_{h}\right)-f_{h}\left(\vec{w}_{h}\right)\right| \leq C h\|\vec{p}\|_{1, q, I}\left\|\vec{w}_{h}\right\|
$$

for al1 $\vec{w}_{\mathrm{h}} \in \vec{V}_{\mathrm{h}}$.

Proof :

Observe first that the inclusion with continuous injection $W^{1, q}(I) \Leftrightarrow \mathscr{Q}(I)$ gives sense to the expression (3.3.12). Next, observe that this definition (3.3.12)'is obtained from (1.2.4) by using the following numerical integration scheme over the reference interval $[0,1]$ :

$$
\int_{0}^{1} \hat{\phi}(\hat{\xi}) \mathrm{d} \hat{\xi} \sim \frac{1}{2}[\hat{\phi}(0)+\hat{\phi}(1)] .
$$

Since this scheme is in particular exact for constants, the estimate (4.3.1) follows from results of [1, part. III, Theorem 4.4.2] or [2, Theorem 4.2].

Remark 4.3.1 : In particular, the proof of Theorem 4.3.1 shows that the definition of $f_{h}$ through the scheme (4.3.2) can be weakened. For instance, we could use the scheme

$$
\int_{0}^{1} \hat{\phi}(\hat{\xi}) d \hat{\xi} \sim \hat{\phi}\left(\frac{1}{2}\right)
$$


which is exact for constants.

4.4 - The bilinear form $\vec{b}_{h}(.,$.$) is uniformly \vec{V}_{h}-$ elliptic

In Theorem 4.1.1. which gives the abstract error estimate, we have assumed the property $(4.1 .1)$, i.e., the bilinear form $b_{h}(.,$.$) is uniformly \vec{V}_{h}$ - elliptic. In the next theorem, we check that this property is effectively satisfied.

Theorem 4.4.1.: Assume that the numerical integration schemes which appear in $(3.3 .11)(4.2 .47)(4: 2.48)$ are derived from schemes defined over the reference element $K$ and such that

$$
\begin{aligned}
& \forall \hat{\phi} \in P_{0}(\hat{K}) \quad, \hat{E}^{1}(\hat{\phi})=0, \\
& \forall \hat{\phi} \in P_{2}(\hat{K}) \quad, \hat{E}^{2}(\hat{\phi})=0,
\end{aligned}
$$

with $\hat{E}^{\alpha}$ defined by $(4: 2: 51)$.

Then, for any regular partition $\mathscr{F}_{\mathrm{h}}$ of $\bar{I}-\operatorname{see}(2.1 .1)$ there exist constants $h_{1}>0$ and $\alpha>0$, independent of $h$, such that for any $h<h_{1}$ we have

$$
a \mid \vec{v}_{h} \|^{2} \leq b_{h}\left(\vec{v}_{h}, \vec{v}_{h}\right), \forall \vec{v}_{h} \in \vec{v}_{h}
$$

\section{Proof :}

The assumptions of Theorem 4.2:5. are satisfied. Then

$$
\left|a\left(\vec{v}_{h}, \vec{v}_{h}\right)-b_{h}\left(\vec{v}_{h}, \vec{v}_{h}\right)\right| \leq \operatorname{ch}\left\|\vec{v}_{h}\right\|^{2}, \forall \vec{v}_{h} \in \vec{v}_{h}
$$


Moreover by analogy, with [3, Theorem 6.1.3],

we can check that the bilinear form $a(.,$.$) is \vec{V}$ - elliptic. Since $\vec{V}_{h} \subset \vec{V}$, there exists a constant $\beta>0$, independent of $h$, such that

$$
a\left(\vec{v}_{h}, \vec{v}_{h}\right) \geq \beta\left\|\vec{v}_{h}\right\|^{2} \quad, \forall \vec{v}_{h} \in \vec{v}_{h}
$$

Combining $(4.4 .4)$ and $(4.4 .5)$, we obtain

$$
b_{h}\left(\vec{v}_{h}, \vec{v}_{h}\right) \geq(B-C h)\left\|\vec{v}_{h}\right\|^{2}, \forall \vec{v}_{h} \in \vec{v}_{h},
$$

and property (4.4.3) follows for h sufficiently small.

\section{5 - Error estimate}

Now we can prove the error estimate theorem.

Theorem 4.5.1. Let $\vartheta_{h}$ be a regular partition of $\bar{I}$ - see (2.1.1) - Let $\overrightarrow{\mathrm{V}}_{\mathrm{h}}, \overrightarrow{\mathrm{V}}_{\mathrm{h}}$ be the associated finite element spaces respectively defined by $(3.2 .16)$ and $(3.2 .17)$.

Assume that the numerical integration schemes which appear in (3.3.11) (4.2.47) (4.2.48) are derived from schemes defined over the reference element $\mathrm{K}$ and such that

$$
\begin{aligned}
& \forall \hat{\phi} \in P_{a}(\hat{K}), \hat{E}^{1}(\hat{\phi})=0, \\
& \forall \hat{\phi} \in P_{2}(\hat{K}), \quad \hat{E}^{2}(\hat{\phi})=0,
\end{aligned}
$$


where $\hat{E}^{\alpha}(\hat{\phi})$ is defined by $(4: 2: 51)$.

Then, if the solution $\vec{u} \in \vec{v}$ of the variational problem

(1.2.5) belongs to the space $H^{2}(I) \times H^{2}(I) \times H^{3}(I)$, if the

components of the loads $p^{1}, p^{3} \in W^{1}, q(I), q \in \mathbb{R}, q \geq 2$, there exist constants $h_{i}>0$ and $C>0$, independent of $h$, such that for any $\dot{h}<h_{1}$ we have

$$
\left\|\vec{u}-\vec{u}_{h}\right\| \leq \operatorname{Ch}\left[\left(\sum_{\alpha=1}^{2}\left\|u_{\alpha}\right\|_{2}^{2}, I+\left\|u_{3}\right\|_{3}^{2}, I\right)^{1 / 2}+\|\vec{p}\|_{1, q, I}\right]
$$

where $\vec{u}_{h} \in \vec{v}_{h}$ is the solution of the discrete problem (3.3.14).

Proof :

We are allowed to apply Theorem 4.1.1. For the first term of the second member of inequality (4.1.2), we remark that the infinimum is bounded from above taking $\vec{v}_{h}=\vec{\pi}_{h} u$, where $\overrightarrow{n_{h}}=\left(\pi_{h_{1}} u_{1}, \pi_{h_{1}} u_{2}, \pi_{h_{2}} u_{3}\right)$ denotes the $\vec{V}_{h}$ - interpolate of the function $\vec{u} \epsilon \vec{v}$. Then, using results of interpolation theory, we find that there exists a constant $C$, independent of $h$, such that

$$
\left\|\vec{u}-\overrightarrow{\pi_{h} u}\right\| \leq \operatorname{Ch}\left(\sum_{\alpha=1}^{2}\left\|u_{\alpha}\right\|_{2}^{2}, I+\left\|u_{3}\right\|_{3}^{2}, I\right)^{1 / 2}
$$

Next, from Theorem 4.2.5., we have

$$
\left|a\left(\pi_{h} \vec{u}_{h}, \vec{w}_{h}\right)-b_{h}\left(\pi_{h} \vec{u}, \vec{w}_{h}\right)\right| \leq C h\left\|\overrightarrow{\pi_{h} \mathbf{u}}\right\|\left\|\vec{w}_{h}\right\|
$$

so that 


$$
\sup _{\vec{w}_{h} \in \vec{V}_{h}} \frac{\left|a\left(\overrightarrow{\pi_{h} u, \vec{w}_{h}}\right)-b_{h}\left(\overrightarrow{\pi_{h} u}, \vec{w}_{h}\right)\right|}{\left\|w_{h}\right\|} \leq C h\left\|\overrightarrow{\pi_{h} u}\right\|
$$

Since the operator of $V_{h_{1}}$ - interpolation (resp. $V_{h_{2}}$ -
interpolation) leaves invariant the spaces $P\left(K_{1}\right)$ $i=1, \ldots, M_{h}\left(\right.$ resp. $\left.P_{1}\left(K_{i}\right)\right)$, we obtain

$$
\left.\left\|\pi_{h_{1}} u_{\alpha}\right\|_{1}, I \leq C\left\|u_{\alpha}\right\|_{I}, I \quad \text { (resp. }\left\|\pi_{h_{2} u_{3} \|_{2}, I} \leq C\right\|_{3} \|_{2}, I\right)
$$

and, hence

$$
\left\|\overrightarrow{\pi_{h} u}\right\| \leq c\|\vec{u}\|
$$

Finally, from Theorem 4.3.1., we obtain

$$
\sup _{\vec{w}_{h} \in \vec{V}_{h}} \frac{\left|f\left(\vec{w}_{h}\right)-f_{h}\left(\vec{w}_{h}\right)\right|}{\left\|\vec{w}_{h}\right\|} \leq \operatorname{ch}\|\vec{p}\|_{1, q, I} .
$$

Error estimate (4.5.3) follows by combining inequalities $(4.1 .2),(4.5 .4)$ to $(4.5 .7)$. 


\section{BIBLIOGRAPHY}

1. BERNADOU, M. : Sur I'analyse numérique du modèle linéaire de coques minces de W.T. KOITER, Thèse d'Etat, Paris VI, Juin 1978.

2. BERNADOU, M. : Convergence of conforming finite element methods for general shell problems. Int. J. Engng. Sc. 18 (1980), 249-276.

3. BERNADOU, M. ; CIARLET, P.G. : Sur l'ellipticité du modèle linéaire de coques de W.T. KOITER. "Computing Methods in Applied. Sciences and Engineering". Lecture Notes in Economics and Mathematical Systems, Vol. 134, pp. 89-136, SpringerVerlag, Berlin 1976.

4. BERNADOU, M. ; DUCATEL, Y. : Approximation of general shell problem by flat plate elements. (To appear).

5. CIARLET, P.G. : The Finite Element Method for Elliptic Problems. NorthHolland (1978).

6. CIARLET, P.G. : On question of existence in shell theory. J. Indian Math. Soc. 40 (1976), 131-143.

7. CLOUGH, R.W. : The finite element method after twenty five years : a personal view. Computers and Structures, 12 (1980), $361-370$.

8. CLOUGH, R.W. ; JOHNSON, C.P. : A finite element approximation for the analysis of thin shells. Int. J. Solids Structures, 4 , $1968,43-60$.

9. CLOUGH, R.W. ; JOHNSON, C.P. : Finite element analysis of arbitrary thin shells, Proceedings ACI Symposium on concrete thin shells, pp. 333-363, New-York, 1970.

10. DAWE, D.J. : Shel1 analysis using a simple facet element. Journal of Strain Analysis, 7, (1972), 266-270.

11. DAWE, D.J. : Some high-order elements for arches and shelis. In "Finite Elements for Thin Shells and Curved Members", Edited by D.G. ASHWELL and R.H. GALLAGHER, J. Wiley and Sons, London, 1976, pp. 131-153.

12. GELLERT, M. ; LAURSEN, M.E. : Formulation and convergence of a mixed finite element method applied to elastic arches of arbitrary geometry and loading. Comput. Methods Appl. Mech. Engrg., 7, ${ }^{\circ} 3$, (1976), 285-302. 
13. ISHIHARA, $\mathrm{K}_{:}:$A finite element Iumped mass scheme for solving eigenvalue problems of circular arches. Numer. Math. 36, $\mathfrak{n}^{\circ} 3$ (1981), 267-290.

14. JOHNSON, C. : On finite element methods for curved shells using flat elements, in Numerische Behandlung von Differentialgleichungen, pp. 147-154, International Series of Numerical Mathematics, Vol. 27, Birkhaüser Verlag, Basel and Stuttgart, 1975.

15. KIKUCHI, F. : On the validity of an approximation available in the finite element shell analysis. Computers and Structures. 5 $(1975), 1-8$.

16. KIKUCHI, F. : On the validity of the finite element analysis of circular arches represented by an assemblage of beam elements. Comput. Methods App1. Mech. Engrg. $\underline{5}$ (1975), 253-276.

17. KOITER, W.T. : On the nonlinear theory of thin elastic shells. Proc. Kon. Ned. Akad. Wetensch. B 69 (1966), 1-54.

18. KOITER, W.T. : On the foundations of the linear theory of thin elastic shells. Proc. Kon. Ned. Akad. Wetensch., B 73 (1970), 169-195.

19. MOAN, T. : A note on the convergence of finite element approximation for problems formulated in curvilinear coordinate systems. Comput ... Methods App1. Mech. Engrg., 3, (1974), 209-235. 
